\title{
High-order Div- and Quasi Curl-Conforming Basis Functions for Calderón Multiplicative Preconditioning of the EFIE
}

\author{
Felipe Valdés, Francesco P. Andriulli, Kristof Cools, and Eric Michielssen, Fellow, IEEE
}

\begin{abstract}
A new high-order Calderón multiplicative preconditioner (HO-CMP) for the electric field integral equation (EFIE) is presented. In contrast to previous CMPs, the proposed preconditioner allows for high-order surface representations and current expansions by using a novel set of high-order quasi curlconforming basis functions. Like its predecessors, the HO-CMP can be seamlessly integrated into existing EFIE codes. Numerical results demonstrate that the linear systems of equations obtained using the proposed HO-CMP converge rapidly, regardless of the mesh density and of the order of the current expansion.
\end{abstract}

Index Terms - Integral equations, EFIE, high-order basis functions, preconditioning.

\section{INTRODUCTION}

$\mathrm{E}$ LECTRIC field integral equation (EFIE) solvers find widespread use in the analysis of time-harmonic scattering from perfect electrically conducting (PEC) surfaces [1]. This paper presents a new Calderón multiplicative preconditioner (CMP) for the EFIE which, unlike its predecessors, allows for high-order surface representations and current expansions.

The numerical solution of the EFIE requires the discretization of the scatterer's surface in terms of a mesh of planar or curvilinear triangles or quadrangles, and of its current distribution, by means of $N$ vector basis functions. Discretization of the EFIE leads to a dense $N \times N$ system of linear equations in the basis functions' expansion coefficients. The computational cost of iteratively solving this system scales as $O\left(N^{2} N_{\text {iter }}\right)$; here $N^{2}$ is the complexity of multiplying the system matrix with a trial solution vector and $N_{\text {iter }}$ is the number of iterations required for convergence to a

Manuscript received May 17, 2010. This work was supported by the National Science Foundation under Grant DMS 0713771, by the AFOSR STTR Grant "Multiscale Computational Methods for Study of Electromagnetic Compatibility Phenomena" (FA9550-10-1-0180), by the Sandia Grant "Development of Calderón Multiplicative Preconditioners with Method of Moments Algorithms", by the KAUST Grant 399813, and an equipment grant from IBM.

F. Valdés and E. Michielssen are with the Electrical Engineering and Computer Science Department, University of Michigan, Ann Arbor, MI USA. (e-mail: fvaldes@umich.edu)

F. P. Andriulli is with the Politecnio di Torino, Torino 10100, Italy.

$\mathrm{K}$. Cools is with the Department of Information Technology (INTEC), Ghent University, B-9000 Ghent, Belgium.

Color versions of one or more of the figures in this paper are available online at http://ieeexplore.ieee.org. prescribed residual. There exist many "fast methods" that reduce the complexity of a matrix-vector multiplication from $O\left(N^{2}\right)$ to $O(N \log N)$ [2-5]. Often $N_{\text {iter }}$ scales with the condition number of the system matrix, with small condition numbers guaranteeing fast convergence. Unfortunately, the condition number of the EFIE system matrix grows rapidly as the mesh discretization density increases [6]. As a result, the cost of solving the EFIE for structures with subwavelength geometric features often is prohibitively high.

Techniques for preconditioning the EFIE by leveraging Calderón identities have become quite popular in recent years [7-13]. In essence, these techniques exploit the selfregularizing property of the EFIE operator, viz. the fact that the square of the EFIE operator is a compact perturbation of the identity, to produce well-conditioned system matrices even when the mesh includes subwavelength geometric features. Unfortunately, few Calderón preconditioners developed to date are easily integrated into existing codes. The CMP technique proposed in [9] is one of them. The CMP uses two separate discretizations of the EFIE, one in terms of standard Rao-Wilton-Glisson (RWG) basis functions [14], and the other in terms of Buffa-Christiansen (BC) basis functions [15]. The latter are div- and quasi curl-conforming, and geometrically nearly orthogonal to the RWG functions. The effectiveness of the RWG-BC combination in the construction of the CMP stems from the fact that the RWG and BC functions are linked by a well-conditioned Gram matrix and guarantee the annihilation of the square of the discretized hypersingular component of the EFIE operator. We note that Chen and Wilton proposed basis functions similar to the BC ones in the context of analyzing scattering from penetrable objects [16]. Both the BC and Chen-Wilton basis functions are of zeroth-order and designed for use in conjunction with RWG basis functions.

In the last decade, EFIE solvers that use high-order representations of the surface and/or the current density have become increasingly popular. A high-fidelity representation of the surface can be achieved using a high-order parametric mapping from a reference cell to the scatterer surface, usually in the form of curvilinear patches (as opposed to flat ones). Among the many high-order basis functions for representing surface current densities, those proposed by Graglia-WiltonPeterson $(\operatorname{GWP}(p))$ [17], which comprise of products of scalar polynomials (complete up to order $p$ ) and RWG basis 
functions, are very popular. For a given solution accuracy, high-order EFIE solvers have been shown to be more CPU and memory efficient than their zeroth-order counterparts [18]. That said, they still suffer from ill-conditioning when applied to structures with subwavelength geometric features. To allow for a high-order CMP, a high-order extension of the $\mathrm{BC}$ functions is called for. Jan et al. [19] already presented an extension of the $\mathrm{BC}$ basis functions on curvilinear triangular patches; unfortunately their method does not extend to highorder current representations.

This paper presents a true high-order BC extension, viz. a set of high-order div- and quasi curl-conforming functions that, when used in conjunction with the $\operatorname{GWP}(p)$ functions, exhibits the aforementioned properties of the BC-RWG pair. The proposed basis functions are constructed as orthogonal projections of the range of the EFIE operator onto divconforming $\operatorname{GWP}(p) s$ defined on a barycentrically refined mesh; preliminary insights into the construction of these basis functions were presented in [20]. Using these basis functions, a high-order CMP (HO-CMP) is implemented and its effectiveness demonstrated via a suite of numerical examples.

\section{CALDERÓN PRECONDITIONED EFIE AND ITS DISCRETIZATION}

This section describes the CMP EFIE idea. Section II-A describes the standard EFIE and its classical discretization. Section II-B describes the Calderón-preconditioned EFIE along with its CMP discretization.

\section{A. Non-preconditioned EFIE solver}

Consider a closed, simply connected PEC surface $S$ residing in a homogeneous medium with permittivity $\epsilon$ and permeability $\mu$. The (scaled) current density $\boldsymbol{J}$ on $S$ induced by the incident time-harmonic electric field $E^{\text {inc }}$ satisfies the EFIE [21]

$$
\mathcal{T}[\boldsymbol{J}]=-\hat{\boldsymbol{n}}_{r} \times \boldsymbol{E}^{i n c}
$$

where

$$
\mathcal{T}[\boldsymbol{J}]=\mathcal{T}_{s}[\boldsymbol{J}]+\mathcal{T}_{h}[\boldsymbol{J}]
$$

with

$$
\mathcal{T}_{s}[\boldsymbol{J}]=\frac{i k}{4 \pi} \hat{\boldsymbol{n}}_{r} \times \int_{S} \frac{e^{i k\left|\boldsymbol{r}-\boldsymbol{r}^{\prime}\right|}}{\left|\boldsymbol{r}-\boldsymbol{r}^{\prime}\right|} \boldsymbol{J}\left(\boldsymbol{r}^{\prime}\right) d s^{\prime}
$$

and

$$
\mathcal{T}_{h}[\boldsymbol{J}]=\frac{-i}{4 \pi k} \hat{\boldsymbol{n}}_{r} \times \int_{S} \nabla^{\prime} \frac{e^{i k\left|\boldsymbol{r}-\boldsymbol{r}^{\prime}\right|}}{\left|\boldsymbol{r}-\boldsymbol{r}^{\prime}\right|} \nabla_{S}^{\prime} \cdot \boldsymbol{J}\left(\boldsymbol{r}^{\prime}\right) d s^{\prime} .
$$

Here, $k=\omega \sqrt{\epsilon \mu}$ and $\hat{\boldsymbol{n}}_{r}$ is the outward pointing unit vector normal to $S$ at $\boldsymbol{r} ; \omega$ is the angular frequency. A time dependence $e^{-i \omega t}(i=\sqrt{-1})$ is assumed and suppressed. The subscripts " $s$ " and " $h$ " stand for "singular" (vector potential) and "hyper-singular" (scalar potential), respectively. To numerically solve (1), $S$ is approximated by a mesh $S_{\delta}$ of planar or curvilinear triangles with minimum edge size $\delta$, and $\boldsymbol{J}$ is expressed as

$$
\boldsymbol{J}(\boldsymbol{r}) \approx \sum_{j=1}^{N} I_{j} \boldsymbol{f}_{j}(\boldsymbol{r})
$$

where $I_{j}, j=1, \ldots, N$ are expansion coefficients of $\boldsymbol{J}$ in terms of a set of the div-conforming basis functions $F=\left\{\boldsymbol{f}_{j}(\boldsymbol{r}), j=1, \ldots, N\right\}$.

Throughout this paper it is assumed that $F$ is the set of $p^{\text {th }}$ order interpolatory Graglia-Wilton-Peterson functions, i.e. $F=\operatorname{GWP}(p)$ [17]. These functions interpolate at $p+1$ and $p(p+1)$ nodes along each of the $N_{E}$ edges and on each of the $N_{P}$ patches in $S_{\delta}$, respectively; the total number of $\operatorname{GWP}(p)$ functions therefore is $N=(p+1) N_{E}+p(p+1) N_{P}$; note that $\mathrm{RWG}=\mathrm{GWP}(0)$ [17]. $\operatorname{GWP}(p)$ functions that interpolate at a node internal to a patch or on an edge henceforth will be referred to as patch and edge functions, respectively. For later use we note the Euler identity for a simply connected surface

$$
N_{V}-N_{E}+N_{P}=2,
$$

where $N_{V}$ is the number of vertices in $S_{\delta}$.

Substitution of expansion (5) into (1), and testing the resulting equation with curl-conforming functions in $n F=\left\{\hat{\boldsymbol{n}}_{r} \times \boldsymbol{f}_{i}(\boldsymbol{r}), i=1, \ldots, N \mid \boldsymbol{f}_{i}(\boldsymbol{r}) \in F\right\} \quad$ yields the $N \times N$ linear system of equations

$$
\mathbf{T}_{F} \mathbf{I}=\mathbf{V}_{F},
$$

where

$$
\begin{gathered}
\left(\mathbf{T}_{F}\right)_{i, j}=\left\langle\hat{\boldsymbol{n}}_{r} \times \boldsymbol{f}_{i}, \mathcal{T}\left[\boldsymbol{f}_{j}\right]\right\rangle, \\
(\mathbf{I})_{j}=I_{j},
\end{gathered}
$$

and

$$
\left(\mathbf{V}_{F}\right)_{i}=-\left\langle\hat{\boldsymbol{n}}_{r} \times \boldsymbol{f}_{i}, \hat{\boldsymbol{n}}_{r} \times \boldsymbol{E}^{i n c}\right\rangle .
$$

Here $\langle\boldsymbol{a}, \boldsymbol{b}\rangle=\int_{S_{r}} \boldsymbol{a}(\boldsymbol{r}) \cdot \boldsymbol{b}(\boldsymbol{r}) d s$ denotes the inner product between to vector functions $\boldsymbol{a}$ and $\boldsymbol{b}$ on $S_{\delta}$.

When analyzing electromagnetic phenomena involving electrically large and/or complex structures, i.e., when $N$ is large, (7) cannot be solved directly and iterative solvers are called for. The computational cost of solving (7) iteratively is proportional to the cost of multiplying the impedance matrix $\mathbf{T}_{F}$ by a trial solution vector and the number of iterations $N_{\text {iter }}$ required to reach a desired residual error; $N_{\text {iter }}$ typically is proportional to $\mathbf{T}_{F}$ 's condition number, viz. the ratio of $\mathbf{T}_{F} s$ 's largest and smallest singular values. Unfortunately, the singular values of the operator $\mathcal{T}$ comprise two branches, one accumulating at zero, and the other at infinity [6]. Thus the condition number of $\mathbf{T}_{F}$ grows without bound as $\boldsymbol{J}$ is increasingly well-approximated, i.e. as $\delta \rightarrow 0$ and/or $p \rightarrow \infty$. When this happens the number of iterations required for convergence often is prohibitively high.

\section{B. Calderón preconditioned EFIE solver}

A well-conditioned EFIE can be obtained by leveraging $\mathcal{T}$ 's self-regularizing property expressed by the Calderón identity [6,9-10],

$$
\mathcal{T}^{2}[\boldsymbol{J}]=-\frac{\boldsymbol{J}}{4}+\mathcal{K}^{2}[\boldsymbol{J}]
$$

with

$$
\mathcal{K}[\boldsymbol{J}](\boldsymbol{r})=\frac{\hat{\boldsymbol{n}}_{r}}{4 \pi} \times \int_{S} \nabla^{\prime} \frac{e^{i k\left|\boldsymbol{r}-\boldsymbol{r}^{\prime}\right|}}{|\boldsymbol{r}-\boldsymbol{r}|^{\prime}} \times \boldsymbol{J}\left(\boldsymbol{r}^{\prime}\right) d s^{\prime} .
$$


The operator $\mathcal{K}$ is compact on smooth surfaces: its singular values accumulate at zero and the same holds true for $\mathcal{K}^{2}$ [6,22]. It follows that the operator $-1 / 4+\mathcal{K}^{2}$ has a bounded spectrum with singular values accumulating at $-1 / 4$. Eqn. (11) implies that the Calderón-preconditioned EFIE

$$
\mathcal{T}^{2}[\boldsymbol{J}]=-\mathcal{T}\left[\hat{\boldsymbol{n}}_{r} \times \boldsymbol{E}^{\text {inc }}\right]
$$

may be amenable to stable discretization regardless of the mesh density or basis function order.

Unfortunately, the discretization of $\mathcal{T}^{2}[\boldsymbol{J}]=\mathcal{T}[\mathcal{T}[\boldsymbol{J}]]$ is by no means trivial. The literature abounds with techniques for discretizing

$$
\mathcal{T}^{2}[\boldsymbol{J}]=\mathcal{T}_{s}^{2}[\boldsymbol{J}]+\mathcal{T}_{s} \mathcal{T}_{h}[\boldsymbol{J}]+\mathcal{T}_{h} \mathcal{T}_{s}[\boldsymbol{J}]+\mathcal{T}_{h}^{2}[\boldsymbol{J}]
$$

that separately handle the first three terms in the above expansion, explicitly leaving out the fourth as $\mathcal{T}_{h}^{2} \equiv 0$ [7-8]. However, the implementation of these techniques into existing codes is quite intrusive. The CMP proposed in [9] does not suffer from this drawback. The CMP approximates $\mathcal{T}^{2}[\boldsymbol{J}]$ as the product of two impedance matrices $\mathbf{T}_{\tilde{F}}$ and $\mathbf{T}_{F}$ with $\tilde{F}=\left\{\tilde{f}_{j}(\boldsymbol{r}), j=1, \ldots, N\right\}$, separated by a Gram matrix that accounts for the possible lack of (bi-)orthogonality between the functions in $\tilde{F}$ and $n F$. In other words, the CMP matrix equation reads

$$
\mathbf{T}^{\mathrm{CMP}} \mathbf{I}=\mathbf{V}^{\mathrm{CMP}}
$$

where

$$
\begin{gathered}
\mathbf{T}^{\mathrm{CMP}}=\mathbf{T}_{\widetilde{F}} \mathbf{G}_{n F ; \widetilde{F}}^{-1} \mathbf{T}_{F}, \\
\mathbf{V}^{\mathrm{CMP}}=\left(\mathbf{T}_{\widetilde{F}} \mathbf{G}_{n F ; \widetilde{F}}^{-1}\right) \mathbf{V}_{F},
\end{gathered}
$$

and

$$
\left(\mathbf{G}_{n F ; \widetilde{F}}\right)_{i, j}=\left\langle\hat{\boldsymbol{n}}_{r} \times \boldsymbol{f}_{i}, \tilde{\boldsymbol{f}}_{j}\right\rangle
$$

is the matrix of overlap integrals of functions in $\tilde{F}$ and $n F$. Eqn. (15) does not require the decomposition of matrix elements in $\mathbf{T}_{\widetilde{F}}$ and $\mathbf{T}_{F}$ into their singular (vector potential) and hypersingular (scalar potential) components, simplifying its implementation. That said, (15) only will be wellconditioned if

C1. the functions in $\tilde{F}$ and $F$ are div-conforming;

C2. the matrix $\mathbf{G}_{n F ; \widetilde{F}}$ is well-conditioned; this ensures the rapid iterative solution of $\mathbf{G}_{n F ; F} \mathbf{y}=\left(\mathbf{T}_{F} \mathbf{x}\right)$ for trial solution vectors $\mathbf{x}$ while solving $(15)$; this requirement precludes the choice $\tilde{F}=F=\operatorname{GWP}(p)$ as such leads to a singular Gram matrix;

C3. the sets $\tilde{F}$ and $F$ ensure the cancellation of $\mathcal{T}_{h}^{2}[\boldsymbol{J}]$ upon discretization, i.e.

$$
\mathbf{T}_{h, \widetilde{F}} \mathbf{G}_{n F ; \widetilde{F}}^{-1} \mathbf{T}_{h, F}=0
$$

where

$$
\left(\mathbf{T}_{h, F}\right)_{i, j}=\left\langle\hat{\boldsymbol{n}}_{r} \times \boldsymbol{f}_{i}, \mathcal{T}_{h}\left[\boldsymbol{f}_{j}\right]\right\rangle .
$$

If (19) is not satisfied, the desirable spectral properties of $\mathcal{T}^{2}$ will not be inherited by $\mathbf{T}_{\widetilde{F}} \mathbf{G}_{n F ; \widetilde{F}}^{-1} \mathbf{T}_{F}$.

The above criteria are satisfied by the sets $F=\mathrm{RWG}$ and $\tilde{F}=\mathrm{BC}$, the set of (zeroth-order) div- and quasi curlconforming Buffa-Christiansen basis functions, used by all CMP implementations reported to date [9-10,12-13,19].

\section{ZEROTH-ORDER QUASI CURL-CONFORMING BASIS FUNCTIONS}

This section reviews the construction of the $\mathrm{BC}$ basis functions and their main properties $[9-10,15]$.

Just as $F=$ RWG, the set $\tilde{F}=\mathrm{BC}$ contains $N=N_{E}$ basis functions. Contrary to the current of the RWG function $f_{n}$, which crosses edge $n$ (Fig. 1(a)), that of the BC function $\tilde{f}_{n}$ flows along edge $n$ (Fig. 1(c)). Consider the barycentrically refined mesh $\bar{S}_{\delta}$, obtained by adding the three medians to each triangle of the original mesh $S_{\delta}$. Each BC basis function is a linear combination of div-conforming RWGs defined on $\bar{S}_{\delta}[9-10,15]$. Even though BC functions are strictly divconforming, they also are quasi curl-conforming in that they resemble curl-conforming RWGs in $n F$ (Fig. 1(b)). This renders the Gram matrix in (18) (with $F=$ RWG and $\tilde{F}=\mathrm{BC}$ ) well-conditioned. That is, the sets $F=\mathrm{RWG}$ and $\tilde{F}=\mathrm{BC}$ fulfill conditions $\mathrm{C} 1$ and $\mathrm{C} 2$ above. To show that these sets also satisfy condition $\mathrm{C} 3$, consider the space $\operatorname{Span}\left(F^{\text {sol }}\right) \subset \operatorname{Span}(F) \quad$ spanned by "div-conforming solenoidal RWG" functions

$$
F^{\text {sol }}=\left\{\boldsymbol{f}_{j}^{\text {sol }}(\boldsymbol{r}), j=1, \ldots, N^{\text {sol }}\right\}
$$

with $N^{\text {sol }}=N_{V}-1$; the $f_{j}^{\text {sol }}$ are charge-free and could, for example, be "loop" functions describing current flowing around all but one of the vertices in $S_{\delta}$ (Fig. 2(a)) [23-24]. The set $F^{\text {sol }}$ can be complemented by a set $F^{\text {nonsol }}$ such that $\operatorname{Span}(F)=\operatorname{Span}\left(F^{\text {sol }}\right) \oplus \operatorname{Span}\left(F^{\text {nonsol }}\right)$. The set $F^{\text {nonsol }}$ contains "div-conforming non-solenoidal RWG" functions

$$
F^{\text {nonsol }}=\left\{\boldsymbol{f}_{j}^{\text {nonsol }}(\boldsymbol{r}), j=1, \ldots, N^{\text {nonsol }}\right\}
$$

with $N^{\text {nonsol }}=N-\left(N_{V}-1\right)=N_{P}-1$; the $\boldsymbol{f}_{j}^{\text {nonsol }}$ all produce charge and could, for example, be "star" functions describing current flowing out of all but one patch in $S_{\delta_{\tilde{F}}}$ (Fig. 2(b)) [2324]. Similarly, consider the space $\operatorname{Span}\left(\tilde{F}^{\text {sol }}\right) \subset \operatorname{Span}(\tilde{F})$ spanned by "div-conforming solenoidal BC" functions

$$
\tilde{F}^{s o l}=\left\{\tilde{\boldsymbol{f}}_{j}^{\text {sol }}(\boldsymbol{r}), j=1, \ldots, \tilde{N}^{\text {sol }}=N^{\text {nonsol }}\right\} .
$$

The dimensionality of $\operatorname{Span}\left(\tilde{F}^{\text {sol }}\right)$ equals that of $\operatorname{Span}\left(F^{\text {nonsol }}\right)$; indeed, it can be verified that an appropriate linear combination of the $\mathrm{BC}$ functions associated with the three edges of a patch in $S_{\delta}$ describes a divergence-free current circulating the patch (Fig. 2(c)) [10]. The set $\tilde{F}^{\text {sol }}$ can be complemented by a set $\tilde{F}^{\text {nonsol }}$ such that $\operatorname{Span}(\tilde{F})=\operatorname{Span}\left(\tilde{F}^{\text {sol }}\right) \oplus \operatorname{Span}\left(\tilde{F}^{\text {nonsol }}\right)$. The set $\tilde{F}^{\text {nonsol }}$ contains "div-conforming non-solenoidal BC" functions

$$
\tilde{F}^{\text {nonsol }}=\left\{\tilde{\boldsymbol{f}}_{j}^{\text {nonsol }}(\boldsymbol{r}), j=1, \ldots, \tilde{N}^{\text {nonsol }}=N^{\text {sol }}\right\} .
$$

Again, the dimensionality of $\operatorname{Span}\left(\tilde{F}^{\text {nonsol }}\right)$ equals that of $\operatorname{Span}\left(F^{\text {sol }}\right)$ (Fig. 2(d)) [10].

Next, assume that the matrices $\mathbf{T}_{h, F}, \mathbf{T}_{h, \tilde{F}}$, and $\mathbf{G}_{n F, \tilde{F}}$, are not constructed using the sets $F=\mathrm{RWG}^{h, \tilde{F}}$ and $\tilde{F}=\stackrel{\mathrm{BC}}{\mathrm{C}}$, but instead from $F^{\text {sol }} \cup F^{\text {nonsol }}$ and $\tilde{F}^{\text {nonsol }} \cup \tilde{F}^{\text {sol }}$ with functions in the left and right subset labeled 1 through $N_{V}-1$ and $N_{V}$ through $N$, respectively; note the reverse order of the "sol" and "nonsol" superscripts for functions in $F=\mathrm{RWG}$ and $\tilde{F}=$ BC . It is clear from (20) and (4) that the entries $\left(\mathbf{T}_{h, F}\right)_{i, j}$ and $\left(\mathbf{T}_{h, \tilde{F}}\right)_{i, j}$ vanish when the source function is solenoidal or the test function is irrotational, which implies 


$$
\mathbf{T}_{h, F}=\left(\begin{array}{cc}
\mathbf{0} & \mathbf{0} \\
\mathbf{0} & \mathbf{T}_{h, F^{\text {nonsol }}}
\end{array}\right) \text {, and } \mathbf{T}_{h, \widetilde{F}}=\left(\begin{array}{cc}
\mathbf{T}_{h, \widetilde{F}^{\text {nowsol }}} & \mathbf{0} \\
\mathbf{0} & \mathbf{0}
\end{array}\right)
$$

The blocks in these matrices have dimensions

$$
\left(\begin{array}{ll}
\left(N_{V}-1\right) \times\left(N_{V}-1\right) & \left(N_{V}-1\right) \times\left(N_{P}-1\right) \\
\left(N_{P}-1\right) \times\left(N_{V}-1\right) & \left(N_{P}-1\right) \times\left(N_{P}-1\right)
\end{array}\right) .
$$

Since an irrotational function can be written as the surface gradient of a scalar function $\phi$, and a solenoidal function can be written as the surface curl of a scalar function $\psi$, the inner product of two such functions can be expressed as

$$
\int_{S}\left(\nabla_{S} \phi(\boldsymbol{r})\right) \cdot\left(\hat{\boldsymbol{n}}_{r} \times \nabla_{s} \psi(\boldsymbol{r})\right) d s
$$

which can be transformed by partial integration into

$$
\int_{S} \phi(r) \nabla_{S} \cdot\left(\hat{n}_{r} \times \nabla_{S} \psi(r)\right) d s=0 .
$$

Therefore, the Gram matrix $\mathbf{G}_{n F: \tilde{F}}$ has the form

$$
\mathbf{G}_{n F ; \widetilde{F}}=\left(\begin{array}{ll}
\mathbf{B} & \mathbf{0} \\
\mathbf{C} & \mathbf{D}
\end{array}\right)
$$

and so does its inverse

$$
\mathbf{G}_{n F ; \widetilde{F}}^{-1}=\left(\begin{array}{cc}
\mathbf{B}^{\prime} & \mathbf{0} \\
\mathbf{C}^{\prime} & \mathbf{D}^{\prime}
\end{array}\right) .
$$

From (25) and (30), it is clear that $\mathbf{T}_{h, \widetilde{F}} \mathbf{G}_{n F, \tilde{F}}^{-1} \mathbf{T}_{h, F}=\mathbf{0}$. The fact that the dimension of the solenoidal subspace of the RWG basis functions equals that of the non-solenoidal subspace of the $\mathrm{BC}$ basis functions (and vice-versa), is essential for the CMP technique to work, as it ensures the cancellation of $\mathcal{T}_{h}^{2}[\boldsymbol{J}]$ upon discretization.

\section{HIGH-ORDER QUASI CURL-CONFORMING BASIS FUNCTIONS}

In this section, a set of div- and quasi curl-conforming highorder extensions of the $\mathrm{BC}$ functions is proposed. In the construction of (15)-(18), for any $p \geq 1$, the new set $\tilde{F}$ is meant to be used alongside

$$
F=\mathrm{RWG} \cup F_{h o}=\mathrm{RWG} \cup F_{h o}^{\text {sol }} \cup F_{h o}^{\text {nonsol }} .
$$

Here $F_{h o}$ complements the set of RWG functions such that $\operatorname{Span}(\operatorname{GWP}(p))=\operatorname{Span}(\mathrm{RWG}) \oplus \operatorname{Span}\left(F_{h o}\right)$. The sets

$$
F_{h o}^{\text {sol }}=\left\{\boldsymbol{f}_{h o, j}^{\text {sol }}(\boldsymbol{r}), j=1, \ldots, N_{h o}^{\text {sol }}\right\}
$$

and

$$
F_{h o}^{\text {nonsol }}=\left\{\boldsymbol{f}_{\text {ho, }}^{\text {nonsol }}(\boldsymbol{r}), j=1, \ldots, N_{h o}^{\text {nonsol }}\right\}
$$

span the solenoidal and non-solenoidal subspaces of $\operatorname{Span}\left(F_{h o}\right)$, respectively. Likewise, $\tilde{F}$ will be constructed as

$$
\tilde{F}=\mathrm{BC} \cup \tilde{F}_{h o}=\mathrm{BC} \cup \tilde{F}_{h o}^{\text {nonsol }} \cup \tilde{F}_{h o}^{s o l},
$$

with sets

$$
\tilde{F}_{h o}^{\text {nonsol }}=\left\{\tilde{\boldsymbol{f}}_{h o, j}^{\text {nonsol }}(\boldsymbol{r}), j=1, \ldots, \tilde{N}_{h o}^{\text {nonsol }}\right\}
$$

and

$$
\tilde{F}_{h o}^{s o l}=\left\{\tilde{\boldsymbol{f}}_{h o, j}^{s o l}(\boldsymbol{r}), j=1, \ldots, \tilde{N}_{h o}^{s o l}\right\}
$$

judiciously chosen such that system (15) is well-conditioned.

Throughout this section, notation introduced previously for spaces and sets applicable to $F$ will be reused and extended for all spaces and functions derived from the barycentrically refined mesh $\bar{S}_{\delta}$ by adding bars on top of symbols. That is,

$$
\bar{F}=\overline{\mathrm{RWG}} \cup \bar{F}_{h o}=\overline{\mathrm{RWG}} \cup \bar{F}_{h o}^{\text {sol }} \cup \bar{F}_{h o}^{\text {nonsol }} \text {, }
$$

where the sets

$$
\bar{F}_{h o}^{s o l}=\left\{\overline{\boldsymbol{f}}_{h o, j}^{s o l}(\boldsymbol{r}), j=1, \ldots, \bar{N}_{h o}^{s o l}\right\}
$$

and

$$
\bar{F}_{h o}^{\text {nonsol }}=\left\{\overline{\boldsymbol{f}}_{h o, j}^{\text {sol }}(\boldsymbol{r}), j=1, \ldots, \bar{N}_{h o}^{\text {nonsol }}\right\}
$$

span the solenoidal and non-solenoidal subspaces of $\operatorname{Span}\left(\bar{F}_{h o}\right)$, respectively; $\overline{\mathrm{GWP}(p)}$ and $\overline{\mathrm{RWG}}$ denote $\operatorname{GWP}(p)$ and RWG basis functions defined on $\bar{S}_{\delta}$; etc.

To guarantee that system (15) has a low condition number, sets $\tilde{F}$ and $F$ must satisfy the above conditions $\mathrm{C} 1$ through C3. To ensure functions in $\tilde{F}$ are div-conforming, they will be constructed as linear combinations of the div-conforming functions in $\bar{F}$. Bases for the high-order solenoidal and nonsolenoidal subspaces $\operatorname{Span}\left(\tilde{F}_{h o}^{\text {sol }}\right)$ and $\operatorname{Span}\left(\tilde{F}_{h o}^{\text {nonsol }}\right)$ will be built separately. To arrive at a well-conditioned Gram matrix $\mathbf{G}_{n F, \tilde{F}}$, functions in $\tilde{F}_{h o}^{\text {sol }}$ ( $\tilde{F}_{h o}^{\text {nonsol }}$ ) will be constructed so as to "resemble" those in $n F_{h o}^{\text {nonsol }}\left(n F_{h o}^{\text {sol }}\right)$. To ensure the cancellation $\mathbf{T}_{h, \tilde{F}} \mathbf{G}_{n F ; \widetilde{F}}^{-1} \mathbf{T}_{h, F}=0$, the cardinality of $\tilde{F}_{h o}^{\text {sol }}$ $\left(\tilde{F}_{h o}^{\text {nonsol }}\right)$ will be matched to that of $F_{h o}^{\text {nonsol }}\left(F_{h o}^{\text {sol }}\right)$ i.e., $\tilde{N}_{h o}^{\text {sol }}=N_{h o}^{\text {nonsol }}$ and $\tilde{N}_{h o}^{\text {nonsol }}=N_{h o}^{\text {sol }}$.

Section IV-A details the Helmholtz decomposition of the spaces $F_{h o}, \bar{F}_{h o}$, and $\tilde{F}_{h o}$. Section IV-B explains how to construct the $\tilde{F}_{h o}$ basis functions such that the Gram matrix $\mathbf{G}_{n F, \tilde{F}}$ is well-conditioned.

\section{A. Helmholtz decomposition}

As described in [25], bases for $\operatorname{Span}\left(F_{h o}^{\text {sol }}\right)$ and $\operatorname{Span}\left(F_{h o}^{\text {nonsol }}\right)$ can be constructed by separating $\operatorname{Span}\left(F_{h o}\right)$ into edge and (internal-to-) patch subspaces. One way of constructing these subspaces is as follows:

1. For each patch in $S_{\delta}$;

a. Define $\mathbf{A}$ as the matrix that maps all $p(p+1)$ patch $\operatorname{GWP}(p)$ functions (columns of $\mathbf{A}$ ) onto their charges (divergence) at points in the patch (rows of A )

b. Perform a singular value decomposition (SVD) on $\mathbf{A}$ : $\mathbf{A}=\mathbf{U} \boldsymbol{\Sigma} \mathbf{V}^{\mathrm{T}}$.

c. The last $p(p-1) / 2$ columns of $\mathbf{V}^{\mathrm{T}}$ are associated with zero singular values, and they describe patch solenoidal functions (Fig. 3(a)).

d. All other columns of $\mathbf{V}^{\mathrm{T}}$ describe patch nonsolenoidal functions (Fig. 3(b)).

2. For each edge in $S_{\delta}$;

a. Define $\mathbf{A}$ as the matrix that maps $p+1$ edge $\operatorname{GWP}(p)$ and the $2 p(p+1)$ overlapping patch $\operatorname{GWP}(p)$ functions (columns of $\mathbf{A}$ ) onto their charges at points in the patches (rows of $\mathbf{A}$ ).

b. Define $\mathbf{B}=\mathbf{A}+\beta \sum \mathbf{u}_{j} \mathbf{v}_{j}^{\mathrm{T}}$, where $\beta$ is a non-zero constant, and $\mathbf{u}_{j}$ and $\mathbf{v}_{j}$ are the singular vectors associated with the patch solenoidal functions identified in step 1 for the two patches that share the edge. Note that the patch solenoidal functions are now associated with singular values equal to $\beta$.

c. Perform a SVD on $\mathbf{B}: \mathbf{B}=\mathbf{U} \boldsymbol{\Sigma} \mathbf{V}^{\mathrm{T}}$. 
d. The last $p$ columns of $\mathbf{V}^{\mathrm{T}}$ are associated with zero singular values, and they describe edge solenoidal functions (Fig. 3(c)).

To summarize, the set $F_{h o}^{s o l}$ contains:

(i) $\quad p(p-1) N_{P} / 2$ high-order patch functions

(ii) and $p N_{E}$ high-order edge functions.

Likewise, $F_{h o}^{\text {nonsol }}$ contains $((p+2)(p+1)-2) N_{P} / 2$ highorder patch functions. The cardinalities of $F_{h o}^{\text {sol }}$ and $F_{h o}^{\text {nonsol }}$ are therefore

$$
N_{h o}^{s o l}=\frac{p(p-1)}{2} N_{P}+p N_{E},
$$

and

$$
N_{h o}^{\text {nonsol }}=((p+2)(p+1)-2) N_{P} / 2 .
$$

Of course $N_{h o}^{\text {sol }}+N_{h o}^{\text {nonsol }}=p N_{E}+p(p+1) N_{P}=N-N_{E}$.

Once the high-order solenoidal and non-solenoidal functions have been obtained as described above, they can be (separately) linearly combined to form a more convenient basis for $\operatorname{Span}\left(F_{h o}^{\text {sol }}\right)$ and $\operatorname{Span}\left(F_{h o}^{\text {nonsol }}\right)$, respectively. A partial local orthogonalization can be performed as follows:

1. For each edge in $S_{\delta}$, orthogonalize the solenoidal functions associated with it.

2. For each patch in $S_{\delta}$, separately orthogonalize the solenoidal and non-solenoidal functions.

After this partial orthogonalization has been performed, all functions in $F_{h o}^{\text {nonsol }}$ are orthogonal to one another, but not necessarily orthogonal to any or all functions in $F_{h o}^{s o l}$. Furthermore, among the functions in $F_{h o}^{\text {sol }}$, only those which are patch based are orthogonal to one another, but not necessarily orthogonal to any or all of the edge solenoidal functions.

A full local orthogonalization can also be performed. The difference with respect to the previous one being that now patch-based solenoidal and non-solenoidal functions are orthogonalized altogether. Hence all functions in $F_{h o}^{\text {nonsol }}$ are orthogonal to one another, and also orthogonal to all patch based functions, but not necessarily to any or all edge based functions in $F_{h o}^{\text {sol }}$.

For future use, we define the matrix $\mathbf{L}$ (of size $N \times N_{h o}^{\text {sol }}$ ) that expresses functions in $F_{h o}^{\text {sol }}$ as linear combinations of functions in $\operatorname{GWP}(p)$, i.e. its $j$ th column contains the coefficients obtained for $\boldsymbol{f}_{h o, j}^{\text {sol }}$ after the orthogonalization process. Similarly, the matrix $\mathbf{S}$ (of size $N \times N_{h o}^{\text {nonsol }}$ ) expresses functions in $F_{h o}^{\text {nonsol }}$ as linear combinations of functions in $\operatorname{GWP}(p)$.

Next, consider the barycentric refinement $\bar{S}_{\delta}$ of $S_{\delta}$. As $S_{\delta}$ is simply connected, so is $\bar{S}_{\delta}$; hence $\bar{S}_{\delta}$ has $\bar{N}_{E}=2 N_{E}+6 N_{P}$ edges, $\bar{N}_{V}=N_{V}+N_{E}+N_{P}$ vertices, and $\bar{N}_{P}=6 N_{P}$ patches. The total number of high-order solenoidal and non-solenoidal functions is

$$
\begin{aligned}
\bar{N}_{h o}^{s o l} & =\frac{p(p-1)}{2} \bar{N}_{P}+p \bar{N}_{E} \\
& =\left(3 p^{2}+3 p+1\right) N_{P}+(2 p+1) N_{E}
\end{aligned}
$$

$$
\begin{aligned}
\bar{N}_{h o}^{\text {nonsol }} & =\left(\frac{(p+2)(p+1)}{2}-1\right) \bar{N}_{P} \\
& =3((p+2)(p+1)-2) N_{P}
\end{aligned}
$$

Matrices $\overline{\mathbf{L}}$ and $\overline{\mathbf{S}}$ can be obtained just as described before, operating on the functions in $\operatorname{GWP}(p)$.

Each function in $\tilde{F}_{h o}^{\text {sol }}$ is built as a linear combination of functions in $\bar{F}_{h o}^{s o l}$, i.e.

$$
\tilde{\boldsymbol{f}}_{h o, j}^{s o l}(\boldsymbol{r})=\sum_{k=1}^{\bar{N}^{s o l}} p_{k, j}^{s o l} \overline{\boldsymbol{f}}_{h o, k}^{s o l}(\boldsymbol{r}), j=1, \ldots, N_{h o}^{\text {nonsol }},
$$

Likewise, each function in $\tilde{F}_{h o}^{\text {nonsol }}$ is built as a linear combination of functions in $\bar{F}_{h o}^{\text {nonsol }}$, i.e.

$$
\tilde{\boldsymbol{f}}_{h o, j}^{\text {nonsol }}(\boldsymbol{r})=\sum_{k=1}^{\bar{N}_{\text {nomosol }}} p_{k, j}^{\text {nonsol }} \overline{\boldsymbol{f}}_{h o, k}^{\text {nonsol }}(\boldsymbol{r}), j=1, \ldots, N_{h o}^{\text {sol }} .
$$

Note that the cardinality of $\tilde{F}_{h o}^{\text {sol }}\left(\tilde{F}_{h o}^{\text {nonsol }}\right.$ ) matches that of $F_{h o}^{\text {nonsol }}\left(F_{h o}^{\text {sol }}\right)$, thereby ensuring the cancellation $\mathbf{T}_{h, \widetilde{F}} \mathbf{G}_{n F ; \widetilde{F}}^{-1} \mathbf{T}_{h, F}=\mathbf{0}$.

\section{B. Well-conditioning of the Gram matrix}

Due to the way the sets $F$ and $\tilde{F}$ are defined, the Gram matrix $\mathbf{G}_{n F: \widetilde{F}}$ can be decomposed into four blocks

$$
\mathbf{G}_{n F ; \widetilde{F}}=\left(\begin{array}{cc}
\mathbf{G}_{n \mathrm{RWG} ; \mathrm{BC}} & \mathbf{G}_{n \mathrm{RWG} ; \widetilde{F} h o} \\
\mathbf{G}_{n F_{h o} ; \mathrm{BC}} & \mathbf{G}_{n F_{h o}, \tilde{F} h o}
\end{array}\right),
$$

where $\mathbf{G}_{n \mathrm{RWG} ; \mathrm{BC}}, \mathbf{G}_{n \mathrm{RWG} ; \widetilde{F}_{h o}}, \mathbf{G}_{n F_{h o} ; \mathrm{BC}}, \quad$ and $\mathbf{G}_{n F_{F_{0} ; \tilde{F}_{L o}}}$ are matrices of size $N_{E} \times N_{E}, N_{E} \times\left(N-N_{E}\right),\left(N-N_{E}\right) \times N_{E}$, and $\left(N-N_{E}\right) \times\left(N-N_{E}\right)$ respectively. The block $\mathbf{G}_{n \mathrm{RWG}: \mathrm{BC}}$ is nothing but the Gram matrix encountered in the zeroth-order case, and it is of course well-conditioned [9-10]. That said, in order for the block $\mathbf{G}_{n F_{n} ; \tilde{F}_{h o}}$ to be well-conditioned the

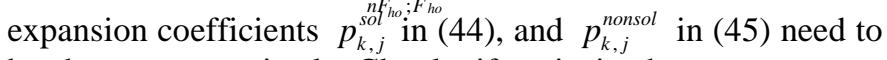
be chosen appropriately. Clearly, if we insist that

$$
\tilde{\boldsymbol{f}}_{h o, j}^{\text {sol }}(\boldsymbol{r}) \approx \hat{\boldsymbol{n}}_{r} \times \boldsymbol{f}_{h o, j}^{\text {nonsol }}(\boldsymbol{r}), j=1, \ldots, N_{h o}^{\text {nonsol }}
$$

and

$$
\tilde{\boldsymbol{f}}_{h o, j}^{\text {nonsol }}(\boldsymbol{r}) \approx \hat{\boldsymbol{n}}_{r} \times \boldsymbol{f}_{h o, j}^{\text {sol }}(\boldsymbol{r}), j=1, \ldots, N_{h o}^{\text {sol }},
$$

then the entries of $\mathbf{G}_{n F_{h}, \tilde{F}_{h o}}$ will be approximately those of $\mathbf{G}_{F_{F_{h}} ; F_{h o}}$. This suggests that the condition number of the former matrix should be similar to that of the latter.

To achieve the resemblance in (47), $\tilde{\boldsymbol{f}}_{h o, j}^{\text {sol }}$ is chosen to be the orthogonal projection of $\hat{\boldsymbol{n}}_{r} \times \boldsymbol{f}_{h o, j}^{\text {nonsol }}$ onto $\operatorname{Span}\left(\bar{F}_{h o}^{s o l}\right)$, i.e.,

$$
\left\langle\overline{\boldsymbol{f}}_{h o, i}^{\text {sol }}, \hat{\boldsymbol{n}}_{r} \times \boldsymbol{f}_{h o, j}^{\text {nonsol }}-\tilde{\boldsymbol{f}}_{h o, j}^{\text {sol }}\right\rangle=0, \forall i=1, \ldots, \bar{N}_{h o}^{\text {sol }} .
$$

Substituting (44) into (49) yields a system of linear equations for the expansion coefficients $p_{k, j}^{\text {sol }}$ :

$$
\sum_{k=1}^{\bar{N}_{k}^{s o l}} p_{k, j}^{s o l}\left\langle\overline{\boldsymbol{f}}_{h o, i}^{s o l}, \overline{\boldsymbol{f}}_{h o, k}^{s o l}\right\rangle=\left\langle\overline{\boldsymbol{f}}_{h o, i}^{s o l}, \hat{\boldsymbol{n}}_{r} \times \boldsymbol{f}_{h o, j}^{\text {nonsol }}\right\rangle, \forall i=1, \ldots, \bar{N}_{h o}^{s o l} .
$$

Eqn. (50) can be expressed in matrix form as

$$
\mathbf{P}_{h o}^{\text {sol }}=\mathbf{G}_{\bar{F}_{h o}^{\text {sol }} ; \bar{F}_{h o}^{\text {sol }}}^{-1} \mathbf{G}_{\bar{F}_{h o}^{\text {sol }} ; n F_{h o}^{\text {nonsol }}}
$$

where the Gram matrices $\mathbf{G}_{\bar{F}_{h o}^{\text {sol }}, \bar{F}_{\text {Fol }}^{\text {sol }}}$ and $\mathbf{G}_{\bar{F}_{h i l}^{\text {sol }} ; n F_{h o}^{\text {nowol }}}$ are

$$
\begin{aligned}
& \left(\mathbf{G}_{\bar{F}_{h o}^{s o l} ; \bar{F}_{h o}^{s o l}}\right)_{i, j}=\left\langle\overline{\boldsymbol{f}}_{h o, i}^{\text {sol }}, \overline{\boldsymbol{f}}_{h o, j}^{\text {sol }}\right\rangle \\
& \left(\mathbf{G}_{\bar{F}_{h o}^{\text {sol }} ; n n_{h o} \text { nonsol }}\right)_{i, j}=\left\langle\overline{\boldsymbol{f}}_{h o, i}^{\text {sol }}, \hat{\boldsymbol{n}}_{r} \times \boldsymbol{f}_{h o, j}^{\text {nonsol }}\right\rangle,
\end{aligned}
$$


and $\left(\mathbf{P}_{h o}^{\text {sol }}\right)_{k, j}=p_{k, j}^{\text {sol }}$. Similarly, enforcing (48) yields the following equation for the expansion coefficients $p_{k, j}^{\text {nonsol }}$ :

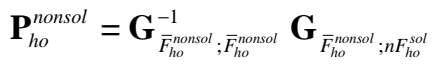

with

$$
\begin{gathered}
\left(\mathbf{G}_{\bar{F}_{h o s}^{\text {nonsol }} ; \bar{F}_{h o}^{\text {nonsol }}}\right)_{i, j}=\left\langle\overline{\boldsymbol{f}}_{\text {ho,i }}^{\text {nonsol }}, \overline{\boldsymbol{f}}_{h o, j}^{\text {nonsol }}\right\rangle, \\
\left(\mathbf{G}_{\bar{F}_{h o}^{\text {nowal }} ; n n_{h o}^{\text {sol }}}\right)_{i, j}=\left\langle\overline{\boldsymbol{f}}_{h o, i}^{\text {nonsol }}, \hat{\boldsymbol{n}}_{r} \times \boldsymbol{f}_{h o, j}^{\text {sol }}\right\rangle,
\end{gathered}
$$

and $\left(\mathbf{P}_{h o}^{\text {nonsol }}\right)_{k, j}=p_{k, j}^{\text {nonsol }}$.

As an example on how these two orthogonal projections perform, consider the div-conforming patch (edge) solenoidal function $\boldsymbol{f}_{h o, j}^{s o l}$ depicted in Fig. 3(a) (Fig. 3(c)). Its curlconforming counterpart $\hat{\boldsymbol{n}}_{r} \times \boldsymbol{f}_{\text {ho, }}^{\text {sol }}$ is shown in Fig. 4(a) (Fig. 4(c)). The orthogonal projection of the latter is the divconforming patch (edge) non-solenoidal function $\tilde{\boldsymbol{f}}_{h o, j}^{\text {nonsol }}$, depicted in Fig. 4(b) (Fig. 4(d)). Similarly, consider the patch non-solenoidal function $\boldsymbol{f}_{h o, j}^{\text {nonsol }}$ depicted in Fig. 3(b). Its curlconforming counterpart $\hat{\boldsymbol{n}}_{r} \times \boldsymbol{f}_{h o, j}^{\text {nonsol }}$ is shown in Fig. 4(e). The orthogonal projection of the latter is the div-conforming patch solenoidal function $\tilde{\boldsymbol{f}}_{h o, j}^{\text {sol }}$ depicted in Fig. 4(f). Note that the support of $\tilde{\boldsymbol{f}}_{h o, j}^{\text {sol }}$ is a couple of "barycentric patches bigger" than the support of $\hat{\boldsymbol{n}}_{r} \times \boldsymbol{f}_{h o, j}^{\text {nonsol }}$. This "extra space" is required as a return path for the current described by $\tilde{\boldsymbol{f}}_{h o, j}^{\text {sol }}$ to provide a charge-free approximation of $\hat{\boldsymbol{n}}_{r} \times \boldsymbol{f}_{h o, j}^{\text {nonsol }}$.

Since the functions in $\tilde{F}_{h o}^{\text {sol }}\left(\tilde{F}_{h o}^{\text {nonol }}\right)$ are built to resemble those in $n F_{h o}^{\text {nonsol }}\left(n F_{h o}^{\text {sol }}\right)$, the condition numbers of $\mathbf{G}_{n F_{h o} ; \widetilde{F}_{h o}}$ and $\mathbf{G}_{n F: \widetilde{F}}$ are expected to depend on the way the functions in $F_{h o}^{s o l}$ and $F_{h o}^{n o n s o l}$ are obtained. Indeed, if functions in $F_{h o}^{s o l}$ and $F_{h o}^{n o n s o l}$ are not orthogonalized in any way described at the end of section IV-A, the condition numbers of $\mathbf{G}_{n F_{h o}, \widetilde{F}_{h o}}$ and $\mathbf{G}_{n F ; \widetilde{F}}$ grow without bound with $p$. As it will be shown later in section VI, partial and full local orthogonalization of the functions in $F_{h o}^{\text {sol }}$ and $F_{h o}^{\text {nonsol }}$ reduce the aforementioned growth on the condition numbers to a minimum. An ideal scenario would be one in which the functions in $F_{h o}^{s o l}$ and $F_{h o}^{n o n s o l}$ are built as one orthogonal set of functions, such that $\mathbf{G}_{F_{h o} ; F_{h o}}$ equals the identity. Hence the matrix $\mathbf{G}_{n F_{F_{0}}: \widetilde{F}_{h o}}$ would be as close as it can be to the identity matrix. Of course, such orthogonalization cannot be performed "locally" therefore it is far from being practical due to its computational cost.

\section{IMPLEMENTATION OF THE HO-CMP}

This section provides details on the construction of the basis functions in $\tilde{F}$ and their use in the HO-CMP. First, explicit expressions for the matrices $\mathbf{P}_{h o}^{\text {sol }}$ and $\mathbf{P}_{h o}^{\text {nonsol }}$ are given in terms of Gram matrices and basis transformations. With these matrices, expressions for $\mathbf{T}_{\widetilde{F}}, \mathbf{G}_{n F, \tilde{F}}$, and $\mathbf{T}_{F}$ are given. Finally, issues relating to computational cost are discussed.

The evaluation of $\mathbf{P}_{h o}^{\text {sol }}$ in (51) requires the computation of

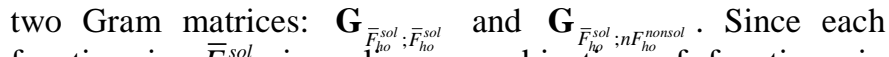
function in $\bar{F}_{h o}^{\text {sol }}$ is a linear combination of functions in $\overline{\operatorname{GWP}(p)}$, the Gram matrix $\mathbf{G}_{\bar{F}_{h o}^{\text {sol }} ; \bar{F}_{h o}^{\text {sol }}}$ in (52) can be obtained as the product

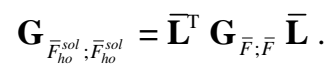

Similarly, the Gram matrix $\mathbf{G}_{\bar{F}_{h o}^{s o l} ; n F_{h o}^{\text {nonsol }}}$ in (53) can be computed as

$$
\mathbf{G}_{\bar{F}_{h o}^{\text {sol }} ; n F_{h o}^{\text {nowsol }}}=\overline{\mathbf{L}}^{\mathrm{T}} \mathbf{G}_{\bar{F} ; n \bar{F}} \mathbf{R} \mathbf{S},
$$

where $\mathbf{R}$ is the matrix (of size $\bar{N} \times N$ ) that expresses functions in $\operatorname{GWP}(p)$ as linear combinations of functions in $\overline{\operatorname{GWP}(p)}$.

The evaluation of $\mathbf{P}_{h o}^{\text {nonsol }}$ in (54) involves the computation

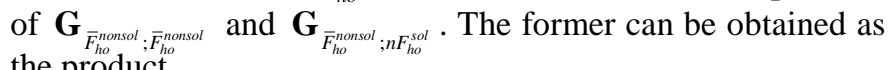
the product

$$
\mathbf{G}_{\bar{F}_{h o}^{\text {nonsol }} ; \bar{F}_{h o}^{\text {nonsol }}}=\overline{\mathbf{S}}^{\mathrm{T}} \mathbf{G}_{\bar{F} ; \bar{F}} \overline{\mathbf{S}} \text {. }
$$

Finally, $\mathbf{G}_{\bar{F}_{h o}^{\text {nonsol }} ; n F_{h o}^{\text {sol }}}$ can be computed as

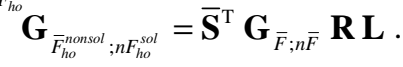

Substitution of the above expressions into eqns. (51) and (54) yields

$$
\begin{aligned}
\mathbf{P}_{h o}^{s o l} & =\left(\overline{\mathbf{L}}^{\mathrm{T}} \mathbf{G}_{\bar{F} ; \bar{F}} \overline{\mathbf{L}}\right)^{-1}\left(\overline{\mathbf{L}}^{\mathrm{T}} \mathbf{G}_{\bar{F} ; n \bar{F}} \mathbf{R} \mathbf{S}\right), \\
\mathbf{P}_{h o}^{\text {nonsol }} & =\left(\overline{\mathbf{S}}^{\mathrm{T}} \mathbf{G}_{\bar{F} ; \bar{F}} \overline{\mathbf{S}}\right)^{-1}\left(\overline{\mathbf{S}}^{\mathrm{T}} \mathbf{G}_{\bar{F} ; n \bar{F}} \mathbf{R} \mathbf{L}\right) .
\end{aligned}
$$

All matrices on the right hand side of (61) and (62) are sparse and can be multiplied by a vector in $O(N)$ operations. Note that the inversion of matrix $\left(\overline{\mathbf{L}}^{\mathrm{T}} \mathbf{G}_{\bar{F}, \bar{F}} \overline{\mathbf{L}}\right)$ need not be performed explicitly, instead its operation on any vector $\mathbf{x}$ can be obtained by solving the linear system $\left(\overline{\mathbf{L}}^{\mathrm{T}} \mathbf{G}_{\bar{F} ; \bar{F}} \overline{\mathbf{L}}\right) \mathbf{y}=\mathbf{x}$ iteratively. Of course, orthogonalization of the functions in $\bar{F}_{h o}^{\text {sol }}$ as described in the previous section makes the matrix well-conditioned. Thus, the evaluation of (61) has an overall computational cost that scales as $O(N)$. Similar considerations apply to the evaluation of (62), with the exception that if the functions in $\bar{F}_{h o}^{\text {nonsol }}$ are orthogonalized, then $\left(\overline{\mathbf{S}}^{\mathrm{T}} \mathbf{G}_{\bar{F} ; \bar{F}} \overline{\mathbf{S}}\right)$ is nothing but the identity, therefore no system need to be solved.

The implementation of the HO-CMP follows the same structure of the zeroth-order CMP (see [9]), which makes use of matrices $\mathbf{P}_{z o}$ and $\mathbf{R}_{z o}$, that express functions in $\mathrm{BC}$ and RWG as linear combinations of functions in $\overline{R W G}$, respectively. The matrix $\mathbf{P}_{z o}$ encountered in the zeroth-order CMP is extended here to $\mathbf{P}$ defined as

$$
\mathbf{P}=\left(\begin{array}{ccc}
\mathbf{P}_{z o} & \mathbf{0} & \mathbf{0} \\
\mathbf{0} & \mathbf{0} & \mathbf{P}_{h o}^{s o l} \\
\mathbf{0} & \mathbf{P}_{h o}^{\text {nonsol }} & \mathbf{0}
\end{array}\right),
$$

where $\mathbf{P}_{h o}^{\text {sol }}$ and $\mathbf{P}_{h o}^{\text {nonsol }}$ are given in (61) and (62) respectively. Explicit expressions for the entries of $\mathbf{P}_{z o}$ can be found in [9]. The matrix $\mathbf{R}_{z o}$ encountered in the zeroth-order CMP is replaced here by the matrix $\mathbf{R}$, defined earlier in this section. Using $\mathbf{P}$, the matrix $\mathbf{T}_{\widetilde{F}}$ in (15) can be discretized as follows:

$$
\mathbf{T}_{\widetilde{F}}=\mathbf{P}^{\mathrm{T}} \mathbf{H}_{\bar{F}}^{\mathrm{T}} \mathbf{T}_{\bar{F}} \mathbf{H}_{\bar{F}} \mathbf{P},
$$

where $\mathbf{H}_{\bar{F}}$ is the matrix that expresses functions in $\bar{F}$ as linear combinations of functions in $\overline{\operatorname{GWP}(p)}$. Similarly, $\mathbf{T}_{F}$ in (15) can be discretized as

$$
\mathbf{T}_{F}=\mathbf{H}_{F}^{\mathrm{T}} \mathbf{R}^{\mathrm{T}} \mathbf{T}_{\bar{F}} \mathbf{R} \mathbf{H}_{F},
$$

where $\mathbf{H}_{F}$ is the matrix that expresses functions in $F$ as linear combinations of functions in $\operatorname{GWP}(p)$.

Finally, with $\mathbf{G}_{n F: \widetilde{F}}$ being discretized as

$$
\mathbf{G}_{n F ; \widetilde{F}}=\mathbf{H}_{F}^{\mathrm{T}} \mathbf{R}^{\mathrm{T}} \mathbf{G}_{n \bar{F} ; \bar{F}} \mathbf{H}_{\bar{F}} \mathbf{P},
$$

the evaluation of (16) and (17) can now be carried out. 
The computational cost of solving (15) is that of multiplying the matrix $\mathbf{T}^{\mathrm{CMP}}$ times the number of iterations required to reach a prescribed residual error. Evaluation of a vector times $\mathbf{T}^{\text {CMP }}$ involves multiplying first by $\mathbf{T}_{F}$ as in (65), then by the inverse of $\mathbf{G}_{n F ; \tilde{F}}$ as in (66), and finally by $\mathbf{T}_{\tilde{F}}$ as in (64). As mentioned previously, the cost of multiplying $\mathbf{R}, \mathbf{P}_{h o}^{\text {sol }}$, and $\mathbf{P}_{h o}^{\text {nonsol }}$ by a vector scales as $O(N)$. Thus, the cost of multiplying $\mathbf{P}$ (and therefore $\mathbf{G}_{n F ; \tilde{F}}$ ) by a vector also scales as $O(N)$. Provided that $\mathbf{G}_{n F: \tilde{F}}$ is well-conditioned, and it is, then its inverse can be multiplied by a vector using just a few (i.e., $O(1)$ ) iterations of an iterative solver like the generalized minimal residual (GMRES) [26] or the transpose-free quasiminimal residual (TFQMR) [27]. Using the multilevel fast multipole method [3], the cost of multiplying $\mathbf{T}_{\bar{F}}$ by a vector scales as $C_{T}+O(N)$ where $C_{T}$ is the cost of multiplying $\mathbf{T}_{F}$ by a vector. Indeed, even though the dimension of $\mathbf{T}_{\bar{F}}$ is greater that that of $\mathbf{T}_{F}$ by a factor of 6 , the additional degrees of freedom introduced by the barycentric mesh do not change the number of multipoles required for field expansion compared to that used when multiplying by $\mathbf{T}_{F}$. Therefore, the cost of multiplying $\mathbf{T}_{\bar{F}}$ increases only by an additive linear term. The fact that the number of iterations required for the HO-CMP to converge is much smaller than that of the standard EFIE justifies the use of the former scheme.

\section{NUMERICAL RESULTS}

This section presents several examples that demonstrate the effectiveness of the basis functions presented in this paper and its performance in the HO-CMP. The results emphasize the main advantage of using a HO-CMP: high-order accuracy in the solutions, without compromising the number of iterations needed for convergence. The results presented here are obtained using a parallel EFIE MoM solver, which uses the proposed HO-CMP or a standard diagonal preconditioner. This solver uses a TFQMR-based iterative method [27] to solve the EFIE MoM systems.

\section{A. High-order accuracy}

The first two examples demonstrate the convergence of the radar cross section (RCS) as the order of the basis functions in the HO-CMP is increased. Each example comprises a smooth PEC object: a sphere of radius $1 \mathrm{~m}$., and a star-shaped object whose surface is parameterized as $r(\theta, \phi)=1.5+\sin ^{2}(2 \theta) \cos ^{2}(\phi) \mathrm{m}$., both illuminated by a 30 MHz., $\hat{x}$-polarized plane wave traveling in the $\hat{z}$ direction. Fig. 5(a) (Fig. 6(a)) shows the bistatic RCS of the PEC sphere (star-shaped object) when computed with basis functions of orders $p=0,1,2,3$. Fig. 5(b) (Fig 6(b)) shows the relative error of the computed RCS of the PEC sphere (star-shaped object) with respect to Mie series ( $4^{\text {th }}$-order) solution. In these examples, the geometric models consist of 32 patches for the sphere and 102 patches for the star-shaped object. Each patch is obtained by means of an exact mapping from a reference patch onto the surface of the object. The evaluation of basis functions on curvilinear patches requires the computation of a Jacobian function, which requires additional computation time when compared to flat patches [17]. The overhead introduced by the evaluation of the Jacobian is more than compensated however by the reduction in the number of patches required to accurately decribe the sphere surface.

\section{B. Condition number}

The following three examples illustrate the behavior of the condition numbers of the non-preconditioned EFIE and HOCMP system matrices as the surface current expansion is increasingly well-approximated, i.e. as $\delta \rightarrow 0$ and/or $p \rightarrow \infty$. Table I shows the condition numbers of $\mathbf{G}_{n F ; \tilde{F}}, \mathbf{T}_{F}$, and $\mathbf{T}^{\mathrm{CMP}}$, obtained with several mesh discretizations of the PEC sphere of Fig. 5(a) using basis functions of orders $p=1,2,3,4$. Similarly, Tables II and III show the same data for the star-shaped object of Fig. 6(a) and a PEC cube with sidelength of $1 \mathrm{~m}$., respectively. These results show that for a fixed order $p$, the condition numbers of $\mathbf{G}_{n F, \tilde{F}}$ and $\mathbf{T}^{\mathrm{CMP}}$ remain bounded as the mesh density is increased, whereas the condition number of $\mathbf{T}_{F}$ does not.

By virtue of the Calderón identity in (11), the operator $\mathcal{T}^{2}$ is spectrally equivalent to the identity operator. Hence the condition number of $\mathbf{T}^{\mathrm{CMP}}$ depends on how well the sets $F$ and $\tilde{F}$ can discretize the identity operator, i.e. the Gram matrix $\mathbf{G}_{n F, \tilde{F}}$. As mentioned in section IV-B, the growth in the condition number of $\mathbf{G}_{n F ; \widetilde{F}}$ (and therefore of $\mathbf{T}^{\mathrm{CMP}}$ ) with $p$ is related to the way in which the functions in $F_{h o}^{s o l}$ and $F_{h o}^{\text {nonsol }}$ are obtained. Table IV shows the condition numbers of $\mathbf{G}_{n F ; \widetilde{F}}$ and $\mathbf{T}^{\mathrm{CMP}}$ for three different ways of obtaining these sets, and for orders $p=1,2,3,4$. As expected, full local orthogonalization of the functions in $F_{h o}^{s o l}$ and $F_{h o}^{\text {sol }}$ result in lower condition numbers for the matrices $\mathbf{G}_{n F ; \tilde{F}}$ and $\mathbf{T}^{\mathrm{CMP}}$ that are more stable with respect to $p$ when compared to partial local orthogonalization. Also, as conjectured at the end of section IV-B, a global orthogonalization of the functions in $F_{h o}^{s o l}$ and $F_{h o}^{\text {sol }}$ yields $\mathbf{G}_{n F ; \tilde{F}}$ and $\mathbf{T}^{\mathrm{CMP}}$ matrices with condition numbers that are almost independent of $p$.

\section{Speed of convergence}

The three examples in this section compare the speed of convergence of the diagonally-preconditioned EFIE and HOCMP when solved iteratively.

Figs. 7(a-e) show the residual error versus iteration count achieved by a TFQMR solver during the iterative solution of the matrix systems obtained by discretizing the diagonallypreconditioned EFIE and HO-CMP with basis functions of orders $p=1,2,3,4,5$. The geometry is a PEC sphere of radius $1 \mathrm{~m}$. Similarly, Figs. 8(a-e) show the same data for a PEC cube with sidelength of $1 \mathrm{~m}$. In both examples, the excitation is a $30 \mathrm{MHz}$., $\hat{x}$-polarized plane wave traveling in the $\hat{z}$ direction, and the prescribed accuracy (relative residual error) for the TFQMR solver is $10^{-5}$. As dictated by the condition number of $\mathbf{T}^{\mathrm{CMP}}$, the number of iterations required for the HO-CMP to reach the prescribed accuracy does not grow as the discretization density is increased. In contrast, the diagonally-preconditioned EFIE requires an increasing number of iterations as the mesh becomes denser. Moreover, this behavior worsens as the order $p$ of the basis functions is 
increased, severely penalizing the efficiency and accuracy of high-order basis functions.

Next, the diagonally-preconditioned EFIE and HO-CMP are used to analyze scattering from a printed monopole antenna similar to the one presented in [28]. The antenna geometry and mesh are shown in Fig. 9(a). Note that the dielectric substrate has not been considered here. The antenna is fed with a voltage delta-gap. The divergence of the electric current, i.e. the (scaled) charge distribution on the surface of the antenna is plotted in Fig. 9(b). The current distribution in this example was obtained using the HO-CMP, with basis functions of order $p=1$ and a frequency of $3.55 \mathrm{GHz}$. The radiation pattern of the antenna is plotted in Fig. 9(c) for two different frequencies: 3.55 and $5.5 \mathrm{GHz}$. Finally, Fig. 9(d) shows the residual error versus iteration count achieved by a TFQMR solver during the iterative solution of the matrix systems stemming from the diagonally-preconditioned EFIE and HOCMP with basis functions of orders $p=0,1$.

The last example involves a model of the Airbus A380 shown in Fig. 10(a). The surface of the aircraft is discretized using second-order curvilinear patches, allowing the use of (relatively) large patches on smooth surfaces (wings and main body), and small patches near fine geometric features (engines and wing tips). The airplane is illuminated by a $\hat{\boldsymbol{y}}$-polarized plane wave traveling in the $\hat{\boldsymbol{x}}$ direction. Fig. 10(b) shows the bistatic RCS obtained for four different frequencies, ranging from 1.5 to $30 \mathrm{MHz}$. Fig. 10(c) and 10(d) show the divergence of the current density induced on the surface of the aircraft, at frequencies of $6 \mathrm{MHz}$ and $30 \mathrm{MHz}$, respectively. Note that at $30 \mathrm{MHz}$ the high-order basis functions allow for the use of less than 5 patches per wavelength on the wings and main body of the aircraft. Finally, Fig. 10(e) shows the residual error versus iteration count achieved by a TFQMR solver during the iterative solution of the matrix systems obtained by discretizing the diagonally-preconditioned EFIE and HO-CMP with basis functions of orders $p=1,2,3$. In this case, the excitation frequency is $6 \mathrm{MHz}$. Similarly, Fig. 10(f) shows the residual error versus iteration count achieved by a TFQMR solver for an excitation frequency of $30 \mathrm{MHz}$. Using basis functions of order $p=0$, it took 30 minutes and 16852 iterations for the diagonally preconditioned EFIE to converge to a prescribed relative residual error of $10^{-4}$. For the HOCMP it took 11 minutes and 485 iterations. Using basis functions of order $p=1$, the diagonally preconditioned EFIE could only reach a relative residual error of $1.8 \times 10^{-3}$ after 8.6 hours and 100000 iterations. For the HO-CMP it took 1.2 hours and 383 iterations to reach the prescribed relative residual error of $10^{-4}$.

\section{CONCLUSION}

In this paper, the CMP technique is extended to high-order by building a set of high-order div- and quasi curl-conforming basis extensions of the $\mathrm{BC}$ basis functions used by all CMP implementations reported to date. Numerical results demonstrate fast convergence rates of the HO-CMP, regardless of the mesh density and the order of the basis functions used. The HO-CMP presented here can be used in the presence of open surfaces with minor modifications. In addition, the basis functions presented here can also be used in high-order Calderón preconditioned formulations for analyzing scattering from penetrable objects.

\section{REFERENCES}

[1] A. J. Poggio, and E. K. Miller, "Integral equation solutions of threedimensional scattering problems," in Computer Techniques for Electromagnetics, R. Mittra, Ed. New York: Pergamon, 1973.

[2] W. C. Chew, J. M. Jin, C. C. Lu, E. Michielssen, and J. M. Song, "Fast solution methods in electromagnetics," IEEE Trans. Antennas Propagat., vol. 45, no. 3, pp. 533-543, Mar. 1997.

[3] J. M. Song, C. C. Lu, and W. C. Chew, "Multilevel fast-multipole algorithm for solving electromagnetic scattering by large complex objects," IEEE Trans. Antennas Propagat., vol. 45, no. 10, pp. 14881493, Oct. 1997.

[4] E. Bleszynski, M. Bleszynski, and T. Jaroszewic, "AIM: Adaptive integral method for solving large-scale electromagnetic scattering and radiation problems," Radio Sci. 31 (1996), pp. 1225-1151.

[5] A. Dutt and V. Rokhlin, "Fast Fourier transforms for nonequispaced data," SIAM J. Sci. Comput. 14 (1993), pp. 1368-1393.

[6] J.-C. Nedéléc, Acoustic and Electromagnetic Equations. New York: Springer-Verlag, 2000.

[7] H. Contopanagos, B. Dembart, M. Epton, J.J. Ottusch, V. Rokhlin, J.L. Visher, and S.M. Wandzura, "Well-conditioned boundary integral equations for three-dimensional electromagnetic scattering," IEEE Trans. Antennas Propagat., vol.50, no.12, pp. 1824-1830, Dec 2002.

[8] R. J. Adams, and N.J. Champagne, "A numerical implementation of a modified form of the electric field Integral equation," IEEE Trans. Antennas Propagat., vol.52, no.9, pp. 2262-2266, Sept. 2004.

[9] F. P. Andriulli, K. Cools, H. Bagci, F. Olyslager, A. Buffa, S. Christiansen, and E. Michielssen, "A Multiplicative Calderón Preconditioner for the Electric Field Integral Equation," IEEE Trans. Antennas Propagat., vol.56, no.8, pp.2398-2412, Aug. 2008.

[10] K. Cools, F.P. Andriulli, F. Olyslager, and E. Michielssen, "TimeDomain Calderón Identities and their Application to the Integral Equations analysis of Scattering by PEC objects Part I: Preconditioning," IEEE Trans. Antennas Propagat., vol. 57, pp. 23522364, Aug 2009.

[11] F. P. Andriulli, and E. Michielssen, "A Regularized Combined Field Integral Equation for Scattering from 2-D Perfect Electrically Conducting Objects," IEEE Trans. Antennas Propagat., vol. 55, pp. 2522-2529, Sept. 2007.

[12] F. Valdés, F.P. Andriulli, H. Bagci, and E. Michielssen, "On the discretization of single source integral equations for analyzing scattering from homogeneous penetrable objects," Antennas and Propagation Society International Symposium, 2008. AP-S 2008. IEEE, vol., no., pp.1-4, 5-11 July 2008.

[13] M. B. Stephanson, and Jin-Fa Lee, "Preconditioned Electric Field Integral Equation Using Calderón Identities and Dual Loop/Star Basis Functions," IEEE Trans. Antennas Propagat., vol. 57, pp. 1274-1279, Apr. 2009.

[14] S. M. Rao, D. R. Wilton, and A W. Glisson, "Electromagnetic scattering by surfaces of arbitrary shape," IEEE Trans. Antennas Propagat., vol. AP-30, pp. 409-418, May 1982.

[15] A. Buffa and S. Christiansen, "A dual finite element complex on the barycentric refinement," Math. Comp., vol. 76, pp. 1743-1769, 2007.

[16] Q. Chen and D. R. Wilton, "Electromagnetic scattering by threedimensional arbitrary complex material/conducting bodies," in Proc. IEEE AP-S Symp., vol. 2, 1990, pp. 590-593.

[17] R.D. Graglia, D.R. Wilton, and A.F. Peterson, "Higher order interpolatory vector bases for computational electromagnetics," IEEE Trans. Antennas Propagat., vol.45, no.3, pp.329-342, Mar 1997.

[18] K. C. Donepudi, J. M. Jin, S. Velamoarambil, J. M. Song, and W. C. Chew, "A higher-order parallelized multilevel fast multipole algorithm for 3D scattering," IEEE Trans. Antennas Propagat., vol. 49, pp. 10781078 , July 2001.

[19] S. Yan, J-M. Jin, and Z. Nie, "Implementation of the Calderón Multiplicative Preconditioner for the EFIE Solution with Curvilinear Triangular Patches," presented in Antennas and Propagation Society International Symposium, 2009. AP-S 2009. IEEE, June 2009. 
[20] F. Valdés, F.P. Andriulli, K. Cools, and E. Michielssen, "High-Order Quasi-Curl Conforming Functions for Multiplicative Calderón Preconditioning of the EFIE," presented in Antennas and Propagation Society International Symposium, 2009. AP-S 2009. IEEE, June 2009.

[21] G. C. Hsiao and R. E. Kleinman, "Mathematical foundations for error estimation in numerical solutions of integral equations in electromagnetics," IEEE Trans. Antennas Propagat., vol. 53, pp. 33163323, Oct. 2005.

[22] R. Kress, Linear Integral Equations, 2nd edition, Springer, New York, 1999.

[23] J.S. Zhao, and W.C. Chew, "Integral equation solution of Maxwell's equations from zero frequency to microwave frequencies," IEEE Trans. Antennas Propagat., vol. 48, pp. 1635-1645, 2000.

[24] W. Wu, A.W. Glisson, and D. Kajfez, "A study of two numerical solutions procedures for the electric field integral equation at low frequency," Appl. Comput. Electromagn. Soc. J., vol. 10, pp. 69-80, 1995.

[25] R.A. Wildman, and D.S. Weile, "An accurate broad-band method of moments using higher order basis functions and tree-loop decomposition," IEEE Trans. Antennas Propagat., vol.52, no.11, pp. 3005-3011, Nov. 2004

[26] Y. Saad, and M.H. Schultz, "GMRES: A generalized minimal residual algorithm for solving non-symmetric linear systems," SIAM J. Sci. Stat. Comput., 3, vol. 7, 1986, 856-869.

[27] R. W. Freund, "A transpose-free quiasi-minimal residual algorithm for non-Hermitian linear systems," SIAM J. Sci. Comput. Volume 14, Issue 2, pp. 470-482, 1993.

[28] M. A. Antoniades, and G. V. Eleftheriades, "A Broadband Dual-Mode Monopole Antenna Using NRI-TL Metamaterial Loading," IEEE Antennas and Wireless Propagation Letters., vol.8, pp. 258-261, May 2009.

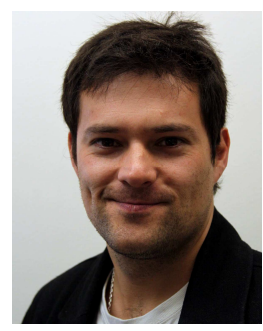

Felipe Valdés received his B.S. degree in electrical engineering from the Pontificia Universidad Católica de Chile, Santiago, Chile, in 2004. Since August 2006 he has been a Research Assistant at the Radiation Laboratory, University of Michigan at Ann Arbor, where he is currently working toward the $\mathrm{Ph} . \mathrm{D}$. degree in electrical engineering. His main research interest is in computational electromagnetics, with focus on preconditioning and single source integral equations.

Mr. Valdés was the recipient of a Fulbright Doctoral Fellowship in 20062010.

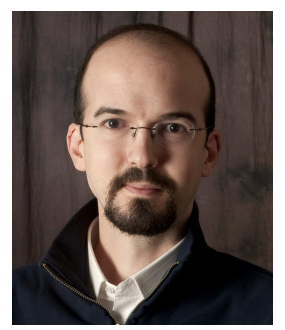

Francesco P. Andriulli (S'05, M'09) received the Laurea degree in electrical engineering from the Politecnico di Torino, Italy, in 2004, the M.S. degree in electrical engineering and computer science from the University of Illinois at Chicago in 2004, and the Ph.D. degree in electrical engineering from the University of Michigan at Ann Arbor in 2008. Since 2008 he has been a Research Associate with the Politecnico di Torino.

His research interests are in computational electromagnetics with focus on preconditioning and fast solution of frequency and time domain integral equations, integral equation theory, hierarchical techniques, and single source integral equations.

Dr. Andriulli was awarded the University of Michigan International Student Fellowship and the University of Michigan Horace H. Rackham Predoctoral Fellowship. He was the recipient of the best student paper award at the 2007 URSI North American Radio Science Meeting. He received the first place prize of the student paper context of the 2008 IEEE Antennas and Propagation Society International Symposium, where he authored and coauthored other two finalist papers. He was the recipient of the 2009 RMTG Award for junior researchers and was awarded a URSI Young Scientist Award at the 2010 International Symposium on Electromagnetic Theory.

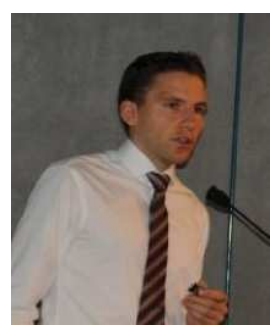

Kristof Cools was born in Merksplas, Belgium, in 1981. He received the M.S degree in physical engineering from Ghent University, Belgium, in 2004. His master's dissertation dealt with the full wave simulation of meta-materials using the low frequency multilevel fast multipole method.

In August 2004, he joined the Electromagnetics Group of the Department of Information Technology (INTEC) at Ghent University. Since then, he has been working towards his $\mathrm{PhD}$ degree under the advisership of Prof. Femke Olyslager (Ghent University) and Prof. Eric Michielssen (University of Michigan). His research focuses on the spectral properties of the boundary integral operators of electromagnetics.

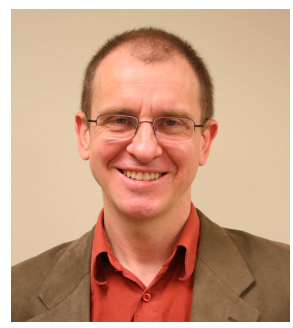

Eric Michielssen (M'95-SM'99-F'02) received his M.S. in Electrical Engineering (Summa Cum Laude) from the Katholieke Universiteit Leuven (KUL, Belgium) in 1987, and his Ph.D. in Electrical Engineering from the University of Illinois at Urbana-Champaign (UIUC) in 1992. He joined the faculty of the UIUC Department of Electrical and Computer Engineering in 1993, reaching the rank of Full Professor in 2002. In 2005, he joined the University of Michigan as Professor of Electrical Engineering and Computer Science. Since 2009, he directs the University of Michigan Computational Science Certificate Program.

Eric Michielssen received a Belgian American Educational Foundation Fellowship in 1988 and a Schlumberger Fellowship in 1990. Furthermore, he was the recipient of a 1994 International Union of Radio Scientists (URSI) Young Scientist Fellowship, a 1995 National Science Foundation CAREER Award, and the 1998 Applied Computational Electromagnetics Society (ACES) Valued Service Award. In addition, he was named 1999 URSI United States National Committee Henry G. Booker Fellow and selected as the recipient of the 1999 URSI Koga Gold Medal. He also was awarded the UIUC's 2001 Xerox Award for Faculty Research, appointed 2002 Beckman Fellow in the UIUC Center for Advanced Studies, named 2003 Scholar in the Tel Aviv University Sackler Center for Advanced Studies, and selected as UIUC 2003 University and Sony Scholar. He is a Fellow of the IEEE (elected 2002) and a member of URSI Commission B.

Eric Michielssen served as the Technical Chairman of the 1997 Applied Computational Electromagnetics Society (ACES) Symposium (Review of Progress in Applied Computational Electromagnetics, March 1997, Monterey, CA), and served on the ACES Board of Directors (1998-2001 and 2002-2003) and as ACES Vice-President (1998-2001). From 1997 to 1999, he was as an Associate Editor for Radio Science, and from 1998 to 2008 he served as Associate Editor for the IEEE Transactions on Antennas and Propagation.

Eric Michielssen authored or co-authored over one 160 journal papers and book chapters and over 280 papers in conference proceedings. His research interests include all aspects of theoretical and applied computational electromagnetics. His research focuses on the development of fast frequency and time domain integral-equation-based techniques for analyzing electromagnetic phenomena, and the development of robust optimizers for the synthesis of electromagnetic/optical devices. 


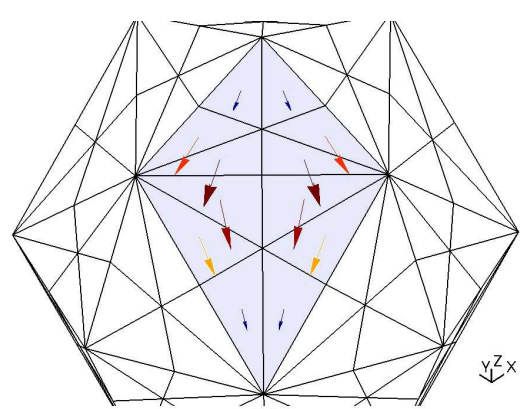

(a)

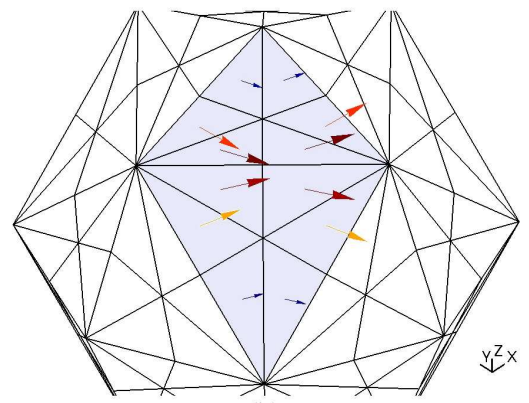

(b)

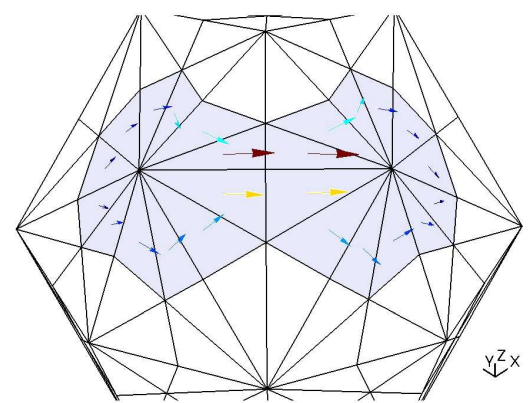

(c)

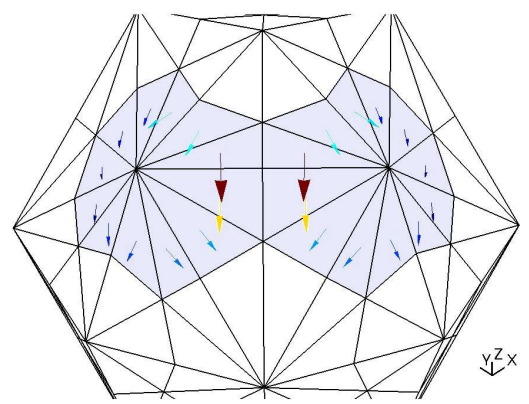

(d)

Fig. 1. RWG and BC functions defined for edge $n$ in $S_{\delta}$. Functions are plotted on top of $\bar{S}_{\delta}$. (a) Div-conforming RWG, $\boldsymbol{f}_{n}$. (b) Curl-conforming RWG, $\hat{\boldsymbol{n}}_{r} \times \boldsymbol{f}_{n}$. (c) Div-conforming BC, $\tilde{\boldsymbol{f}}_{n}$. (d) Curl-conforming BC, $\hat{\boldsymbol{n}}_{r} \times \tilde{\boldsymbol{f}}_{n}$.

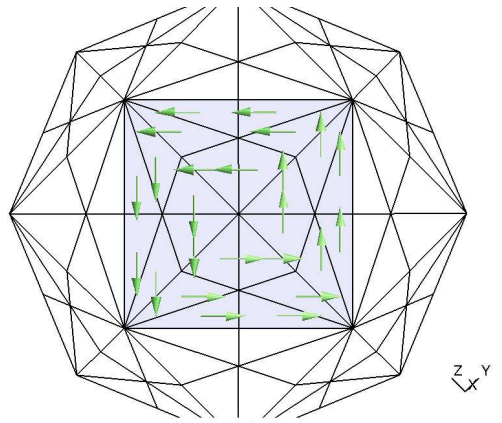

(a)

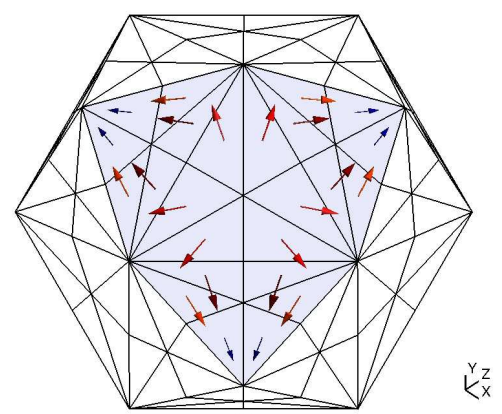

(b)

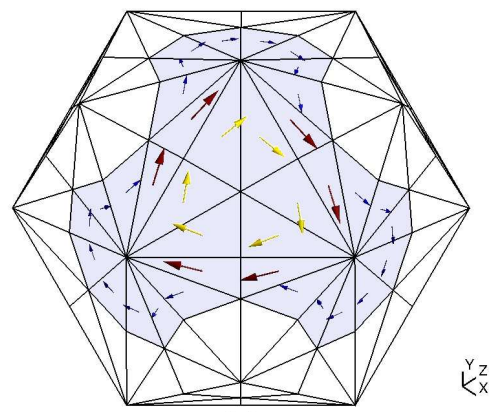

(c)

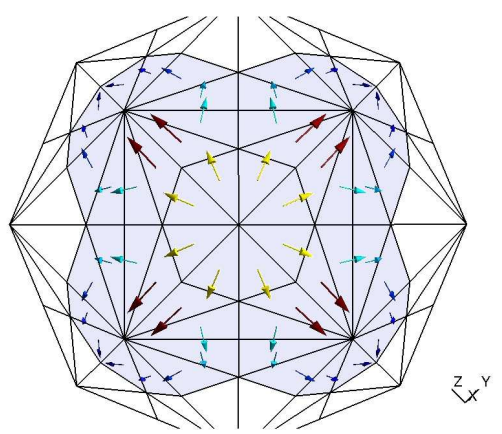

(d)

Fig. 2. Div-conforming RWG and BC solenoidal and non-solenoidal functions defined in $S_{\delta}$. Note that functions are plotted on top of $\bar{S}_{\delta}$. (a) Div-conforming RWG solenoidal function $\boldsymbol{f}_{n}^{\text {sol }}$, describing current flowing around vertex $n$ in $S_{\delta}$. (b) Div-conforming RWG non-solenoidal function $f_{n}^{n o n s o l}$, describing current flowing out of patch $n$ in $S_{\delta}$. (c) Divconforming BC solenoidal function $\tilde{f}_{n}^{\text {sol }}$, describing current flowing around patch $n$ in $S_{\delta}$. (d) Div-conforming BC non-solenoidal function $\tilde{\boldsymbol{f}}_{n}^{\text {nonsol }}$, describing current flowing out of vertex $n$ in $S_{\delta}$. 


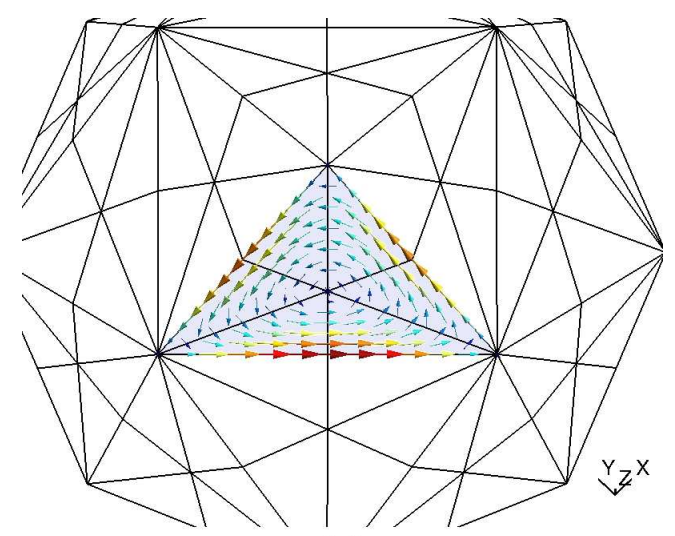

(a)

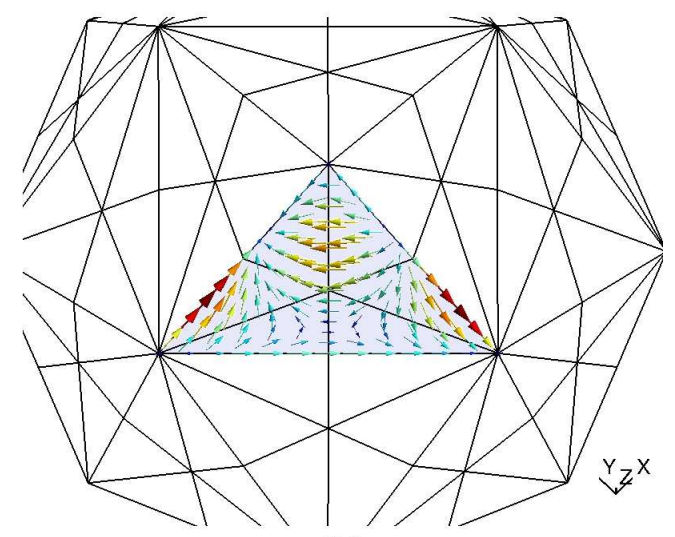

(b)

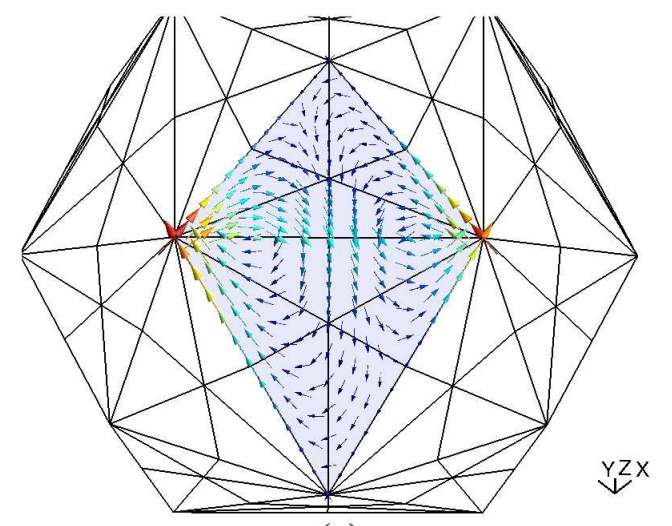

(c)

Fig. 3. Div-conforming $F_{h o}$ solenoidal and non-solenoidal functions defined in $S_{\delta}$. Note that functions are plotted on top of $\bar{S}_{\delta}$. (a) Div-conforming $F_{h o}$ patch solenoidal function $\boldsymbol{f}_{h o, j}^{\text {sol }}$, its support (shaded area) is limited to a patch in $S_{\delta}$. (b) Div-conforming $F_{h o}$ patch non-solenoidal function $\boldsymbol{f}_{h o, j}^{\text {nonsol }}$, its support (shaded area) is limited to a patch in $S_{\delta}$. (c) Div-conforming $F_{h o}$ edge solenoidal function $\boldsymbol{f}_{h o, j}^{\text {sol }}$, its support (shaded area) include the two patches sharing the edge in $S_{\delta}$.
TABLE I

CONDITION NUMBERS OF $\mathbf{G}_{n F ; \tilde{F}}, \mathbf{T}_{F}$, AND $\mathbf{T}^{\text {CMP }}$ FOR THREE DIFFERENT MESH DISCRETIZATIONS OF A PEC SPHERE

\begin{tabular}{|c|c|c|c|c|c|}
\hline \hline$p$ & $N_{P}$ & $N$ & $\mathbf{G}_{n F ; \tilde{F}}$ & $\mathbf{T}_{F}$ & $\mathbf{T}^{\mathrm{CMP}}$ \\
\hline \hline \multirow{3}{*}{1} & 32 & 160 & 11.77 & 335.87 & 21.18 \\
\cline { 2 - 6 } & 102 & 510 & 13.4 & 2318.48 & 25.63 \\
\cline { 2 - 6 } & 224 & 1120 & 14.35 & 5542.08 & 32.95 \\
\hline \hline \multirow{3}{*}{2} & 32 & 336 & 45.06 & 2942.35 & 63.31 \\
\cline { 2 - 6 } & 102 & 1071 & 68.08 & 21298.59 & 98.67 \\
\cline { 2 - 6 } & 224 & 2352 & 59.97 & 47297.28 & 88.26 \\
\hline \hline \multirow{3}{*}{3} & 32 & 576 & 62.59 & 25681.78 & 202.45 \\
\cline { 2 - 6 } & 102 & 1836 & 72.94 & 189540.53 & 233.97 \\
\cline { 2 - 6 } & 224 & 4032 & 78.02 & 417912.93 & 265.26 \\
\hline \hline \multirow{3}{*}{4} & 32 & 880 & 156.68 & 201766.16 & 571.16 \\
\cline { 2 - 6 } & 102 & 2805 & 183.08 & 1604161.36 & 705.08 \\
\cline { 2 - 6 } & 224 & 6160 & 192.28 & 3394907.21 & 740.86 \\
\hline \hline
\end{tabular}

\section{TABLE II}

CONDITION NUMBERS OF $\mathbf{G}_{n F ; \tilde{F}}, \mathbf{T}_{F}$, AND $\mathbf{T}^{\mathrm{CMP}}$ FOR THREE DIFFERENT MESH DISCRETIZATIONS OF A PEC STAR-SHAPED OBJECT

\begin{tabular}{|c|c|c|c|c|c|}
\hline \hline$p$ & $N_{P}$ & $N$ & $\mathbf{G}_{n F ; \tilde{F}}$ & $\mathbf{T}_{F}$ & $\mathbf{T}^{\mathrm{CMP}}$ \\
\hline \hline \multirow{3}{*}{1} & 32 & 160 & 13.24 & 239.07 & 23.56 \\
\cline { 2 - 6 } & 102 & 510 & 14.49 & 1167.83 & 26.5 \\
\cline { 2 - 6 } & 224 & 1120 & 14.06 & 4013.27 & 28.53 \\
\hline \hline \multirow{3}{*}{2} & 32 & 336 & 51.38 & 2005.19 & 78.68 \\
\cline { 2 - 6 } & 102 & 1071 & 91.44 & 11268.25 & 125.03 \\
\cline { 2 - 6 } & 224 & 2352 & 70.19 & 36240.98 & 109.66 \\
\hline \hline \multirow{3}{*}{3} & 32 & 576 & 82.46 & 17867.56 & 262.67 \\
\cline { 2 - 6 } & 102 & 1836 & 88.62 & 102686.45 & 279.92 \\
\cline { 2 - 6 } & 224 & 4032 & 83.03 & 325654.23 & 262.08 \\
\hline \hline \multirow{3}{*}{4} & 32 & 880 & 179.14 & 145715.16 & 700.56 \\
\cline { 2 - 6 } & 102 & 2805 & 226.84 & 905399.7 & 926.65 \\
\cline { 2 - 6 } & 224 & 6160 & 198.94 & 2689593.01 & 735.11 \\
\hline \hline
\end{tabular}

\section{TABLE III}

CONDITION NUMBERS OF $\mathbf{G}_{n F ; \tilde{F}}, \mathbf{T}_{F}$, AND $\mathbf{T}^{\mathrm{CMP}}$ FOR THREE DIFFERENT MESH DISCRETIZATIONS OF A PEC CUBE

\begin{tabular}{|c|c|c|c|c|c|}
\multicolumn{6}{c|}{ MESH DISCRETIZATIONS OF A PEC CUBE } \\
\hline \hline$p$ & $N_{P}$ & $N$ & $\mathbf{G}_{n F: \tilde{F}}$ & $\mathbf{T}_{F}$ & $\mathbf{T}^{\mathrm{CMP}}$ \\
\hline \hline \multirow{3}{*}{1} & 24 & 120 & 14.94 & 658.14 & 26.63 \\
\cline { 2 - 6 } & 154 & 770 & 12.41 & 6719.43 & 35.38 \\
\cline { 2 - 6 } & 240 & 1200 & 12.21 & 10411.1 & 42.66 \\
\hline \hline \multirow{3}{*}{2} & 24 & 252 & 76.75 & 5266.6 & 118.11 \\
\cline { 2 - 6 } & 154 & 1617 & 73.03 & 59766.95 & 115.66 \\
\cline { 2 - 6 } & 240 & 2520 & 59.98 & 97635.17 & 96.78 \\
\hline \hline \multirow{3}{*}{3} & 24 & 432 & 69.48 & 50812.32 & 221.55 \\
\cline { 2 - 6 } & 154 & 2772 & 69.5 & 582413.73 & 315.89 \\
\cline { 2 - 6 } & 240 & 4320 & 71.17 & 841216.68 & 338.87 \\
\hline \hline \multirow{3}{*}{4} & 24 & 660 & 172.38 & 325648.19 & 674.44 \\
\cline { 2 - 6 } & 154 & 4235 & 165.76 & 3910371 & 881.96 \\
\cline { 2 - 6 } & 240 & 6600 & 168.5 & 6382122.93 & 921.35 \\
\hline \hline
\end{tabular}




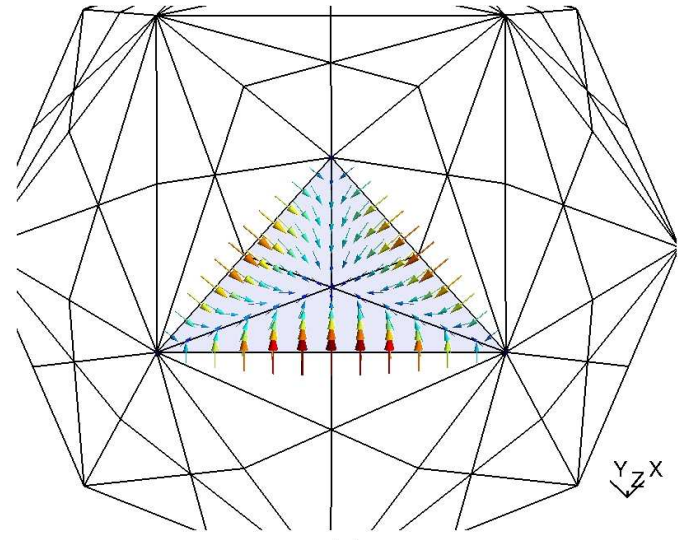

(a)

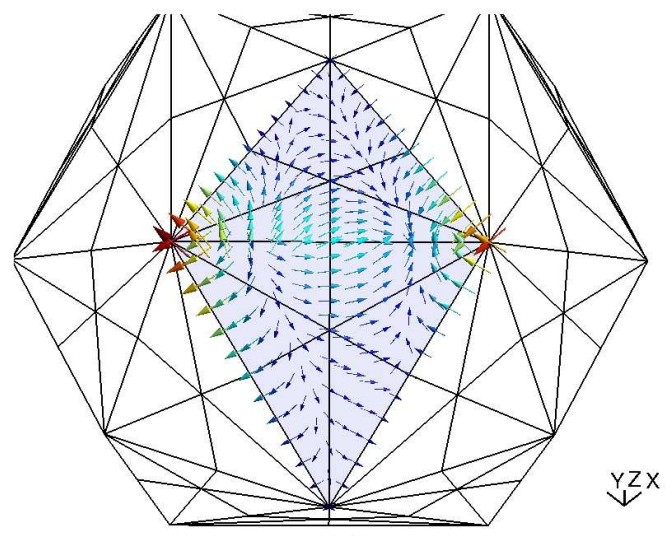

(c)

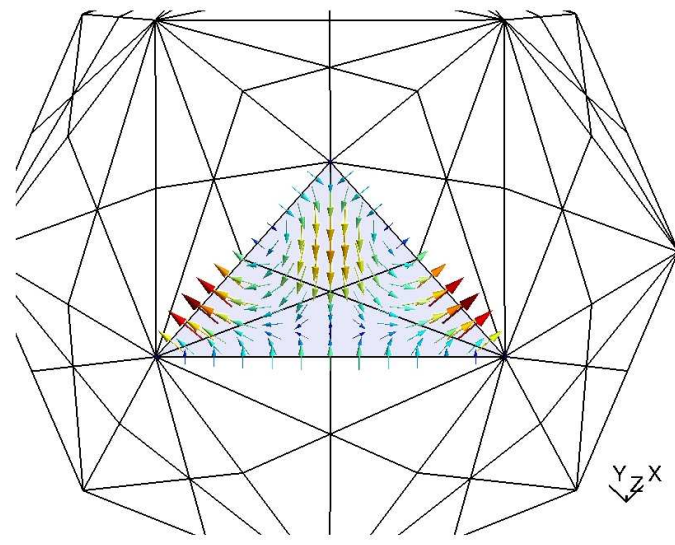

(e)

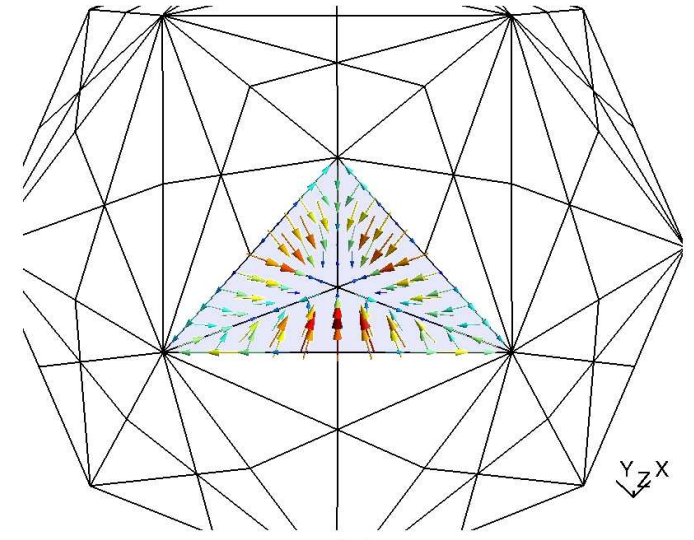

(b)

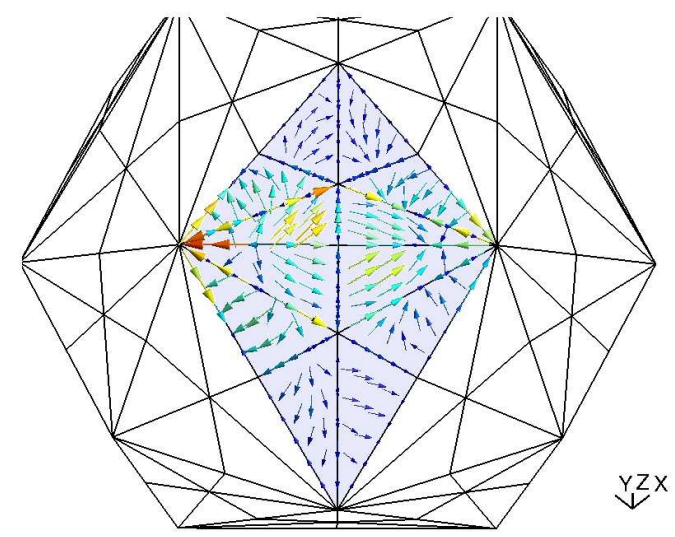

(d)

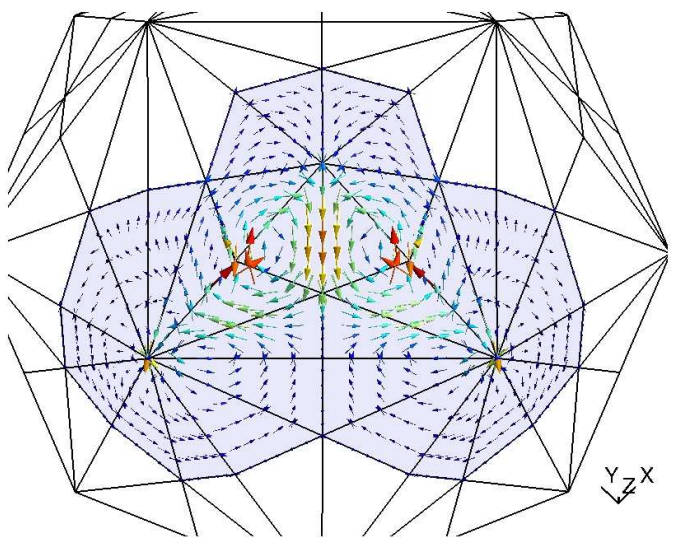

(f)

Fig. 4. Div- and quasi curl-conforming functions in $\tilde{F}_{h o}$, approximating those in $n F_{h o}$. Note that functions are plotted on top of $\bar{S}_{\delta}$. (a) $\hat{\boldsymbol{n}}_{r} \times \boldsymbol{f}_{h o, j}^{\text {sol }}$, i.e. curl-conforming counterpart of the patch solenoidal function $\boldsymbol{f}_{h o, i}^{\text {sol }}$ depicted in Fig.3 (a). (b) Div-conforming patch non-solenoidal function $\tilde{\boldsymbol{f}}_{\text {ho }, j}^{\text {nonsl }}$ approximating $\hat{\boldsymbol{n}}_{r} \times \boldsymbol{f}_{h o, j}^{\text {sol }}$. (c) $\hat{\boldsymbol{n}}_{r} \times \boldsymbol{f}_{h o, j}^{\text {sol }}$, i.e. curl-conforming counterpart of the edge solenoidal function $\boldsymbol{f}_{h o, i}^{\text {sol }}$ depicted in Fig.3 (c). (d) Div-conforming edge non-solenoidal function $\tilde{\boldsymbol{f}}_{h o, j}^{\text {nonsol }}$ approximating $\hat{\boldsymbol{n}}_{r} \times \boldsymbol{f}_{h o, j}^{\text {sol }}$. (e) $\hat{\boldsymbol{n}}_{r} \times \boldsymbol{f}_{h o, j}^{\text {nonsol }}$, i.e. curl-conforming counterpart of the patch non-solenoidal function $\boldsymbol{f}_{h o, j}^{\text {nonsol }}$ depicted in Fig.3 (b). (f) Div-conforming patch solenoidal function $\tilde{\boldsymbol{f}}_{h o, j}^{\text {sol }}$ approximating $\hat{\boldsymbol{n}}_{r} \times \boldsymbol{f}_{h o, j}^{\text {nonsol }}$. 


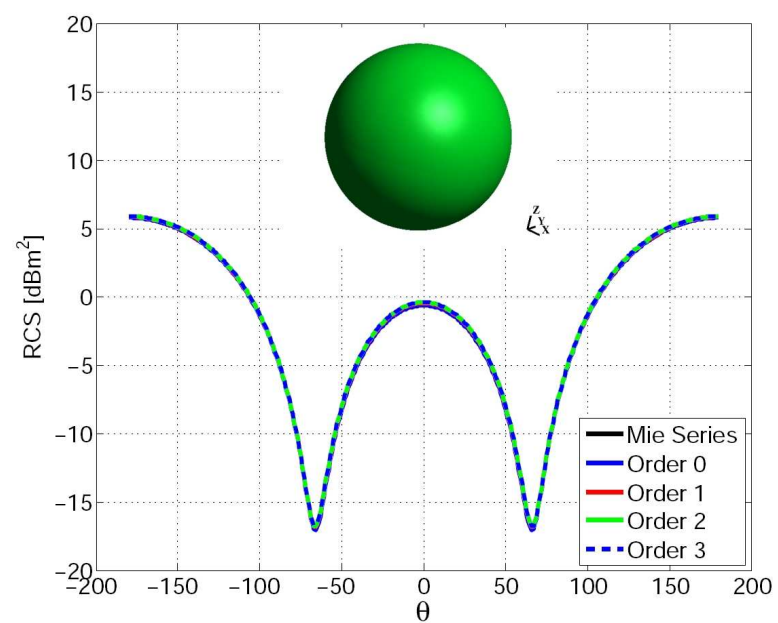

(a)

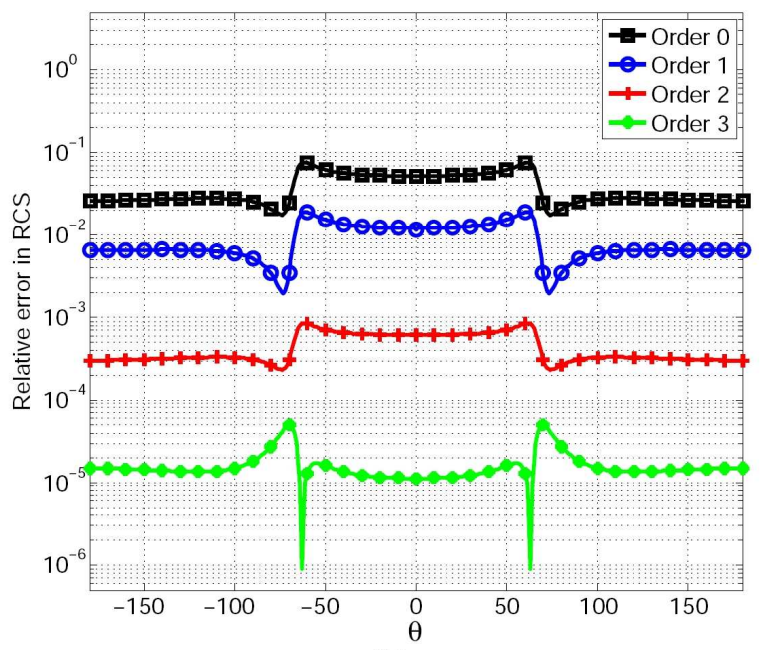

(b)

Fig. 5. Bistatic RCS of a PEC sphere of radius $1 \mathrm{~m}$. illuminated by a 30 $\mathrm{MHz} \hat{x}$-polarized plane wave traveling in the $\hat{z}$ direction. The surface of the sphere is modeled with 32 curvilinear patches. The current density is modeled with basis functions of orders $p=0,1,2,3$. The number of unknowns ranges from $48(p=0)$ to $576(p=3)$ : (a) Bistatic RCS in the $\mathrm{x}$ $\mathrm{z}$ plane. (b) Relative error in the RCS with respect to Mie series solution.

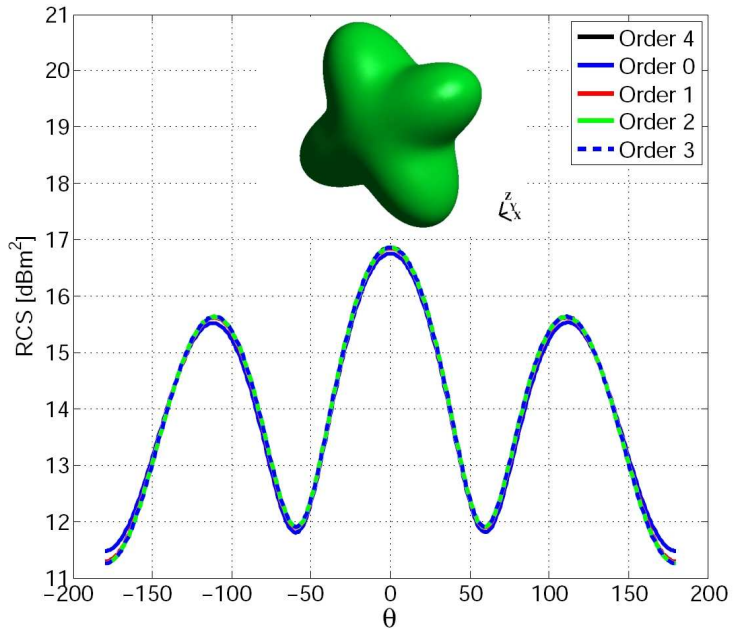

(a)

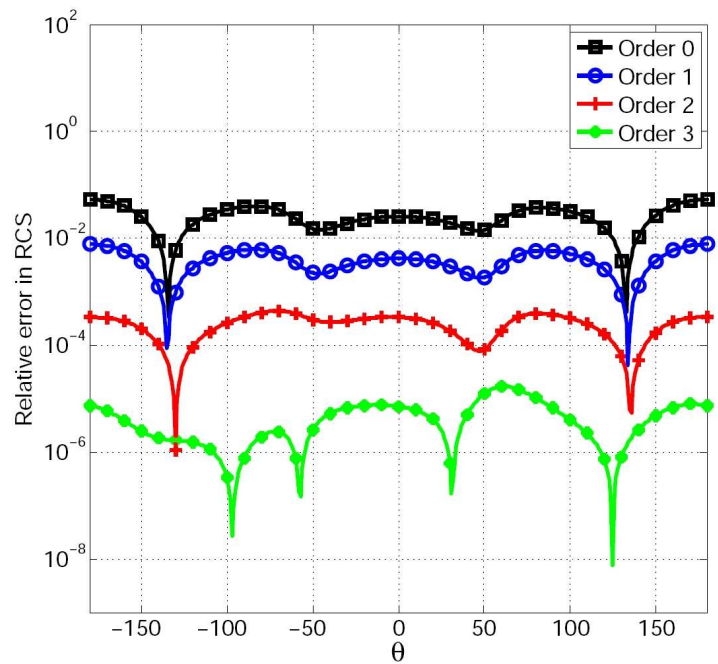

(b)

Fig. 6. Bistatic RCS of a PEC star-shaped object illuminated by a $30 \mathrm{MHz}$ $\hat{x}$-polarized plane wave traveling in the $\hat{z}$ direction. The surface of the object is modeled with 102 curvilinear patches. The current density is modeled with basis functions of orders $p=0,1,2,3$. The number of unknowns ranges from $153(p=0)$ to 1836 ( $p=3$ ). (a) Bistatic RCS in the $\mathrm{x}-\mathrm{z}$ plane. (b) Relative error in the RCS with respect to the solution obtained using basis functions of order $p=4$.

TABLE IV

CONDITION NUMBERS OF $\mathbf{G}_{n F ; \tilde{F}}$, AND $\mathbf{T}^{\text {CMP }}$ FOR THREE DIFFERENT HELMHOLTZ DECOMPOSITION STRATEGIES

\begin{tabular}{|c|c||c|c||c|c||c|c|}
\hline \hline \multicolumn{2}{|c|}{} & \multicolumn{2}{c||}{ Partial local orthogonalization } & \multicolumn{2}{c|}{ Full local orthogonalization } & \multicolumn{2}{c|}{ Full global orthogonalization } \\
\hline \hline$p$ & $N$ & $\mathbf{G}_{n F ; \tilde{F}}$ & $\mathbf{T}^{\mathrm{CMP}}$ & $\mathbf{G}_{n F ; \tilde{F}}$ & $\mathbf{T}^{\mathrm{CMP}}$ & $\mathbf{G}_{n F ; \tilde{F}}$ & $\mathbf{T}^{\mathrm{CMP}}$ \\
\hline \hline 1 & 160 & 29.9 & 21.18 & 29.9 & 20.99 & 2.51 & 3.14 \\
\hline 2 & 336 & 73.1 & 63.31 & 55.5 & 48.15 & 2.54 & 3.17 \\
\hline 3 & 576 & 152.3 & 202.45 & 126.5 & 134.97 & 2.79 & 3.29 \\
\hline 4 & 880 & 220.2 & 571.16 & 177.4 & 248.44 & 2.94 & 3.47 \\
\hline \hline
\end{tabular}




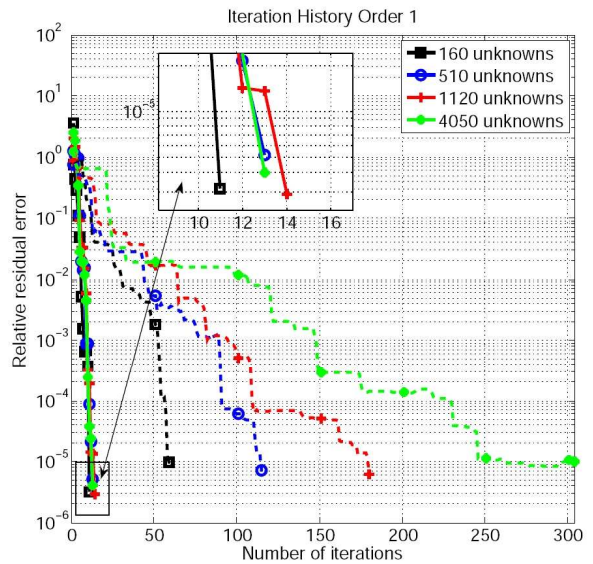

(a)

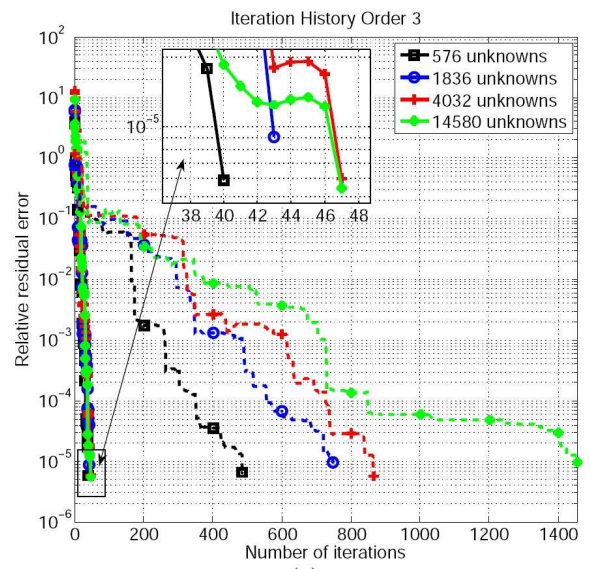

(c)

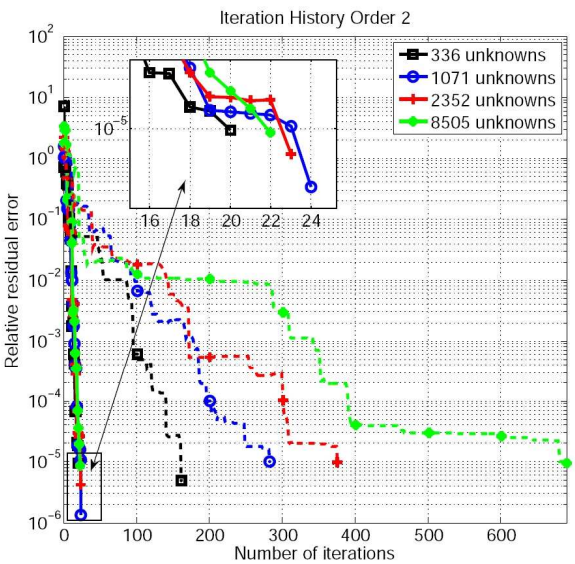

(b)

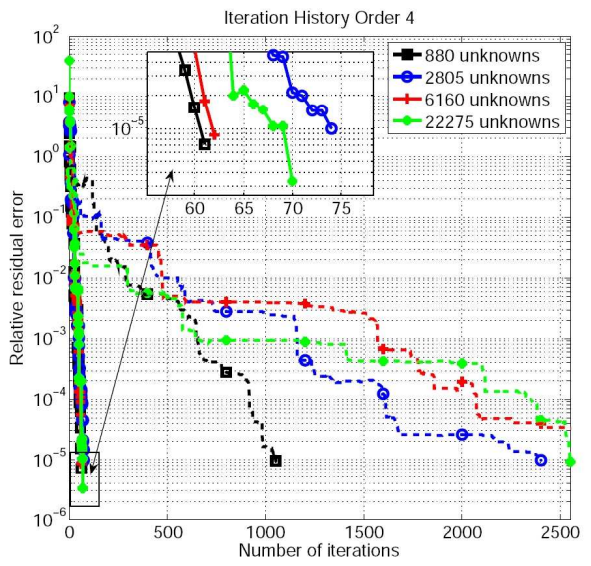

(d)

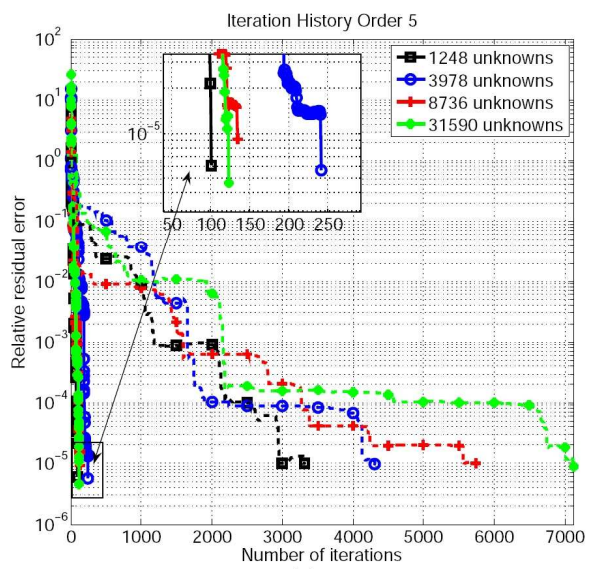

(e)

Fig. 7. Residual history of diagonally-preconditioned EFIE (dashed lines) and HO-CMP (solid lines) for the case of a PEC sphere of radius $1 \mathrm{~m}$., illuminated by a $30 \mathrm{MHz}$., $\hat{x}$-polarized plane wave traveling in the $\hat{z}$ direction. Four different discretizations are used, ranging from 32 to 810 curvilinear elements. Results are shown for several orders of the basis functions: (a) order 1; (b) order 2; (c) order 3; (d) order 4; (e) order 5. 


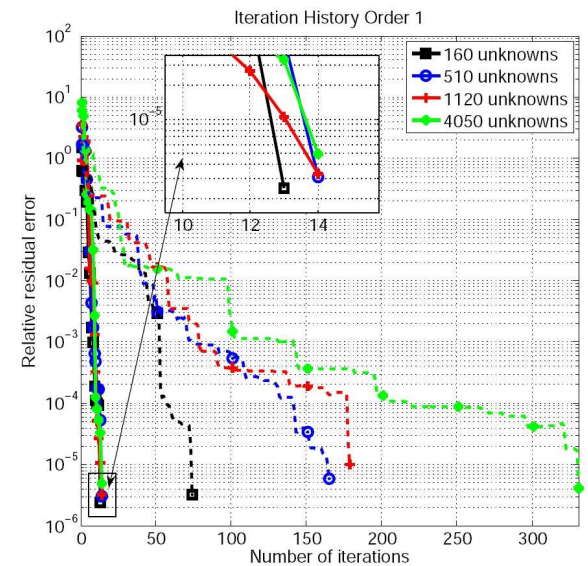

(a)

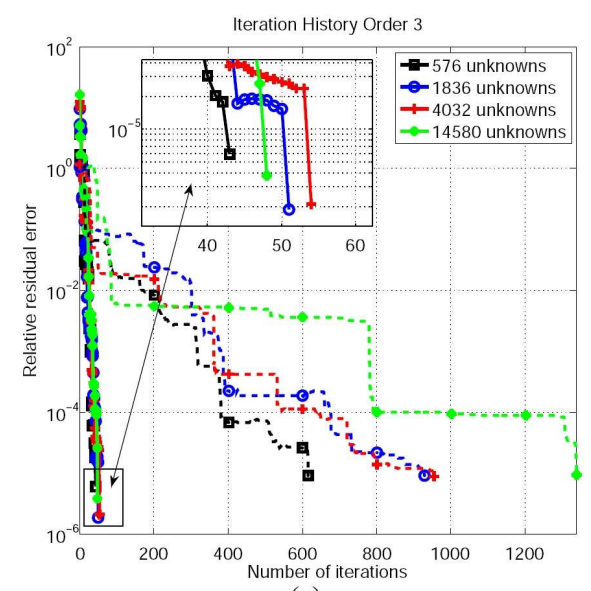

(c)

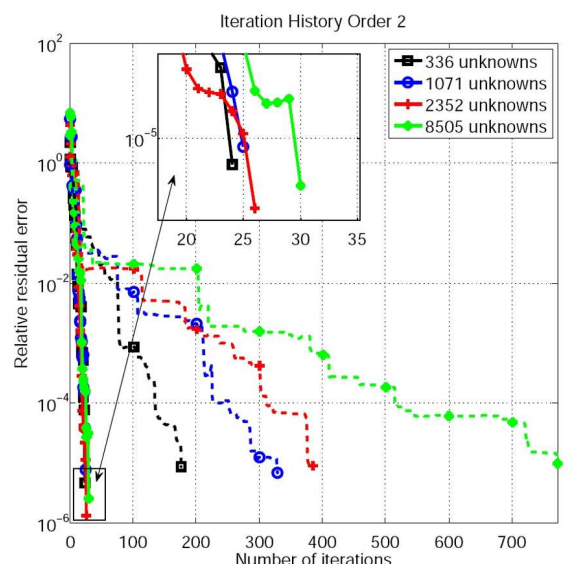

(b)

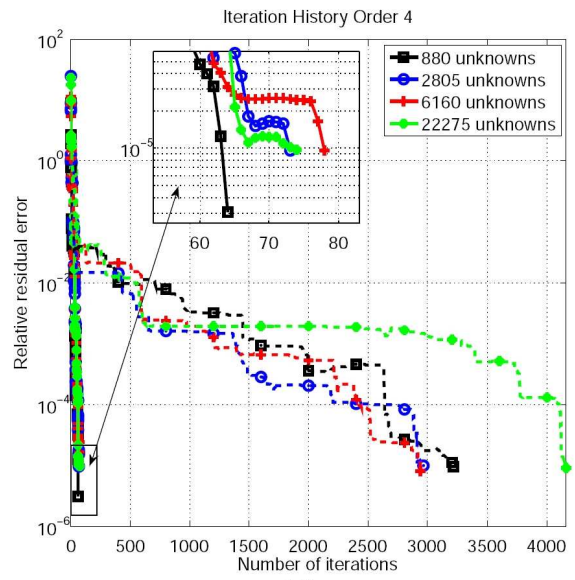

(d)

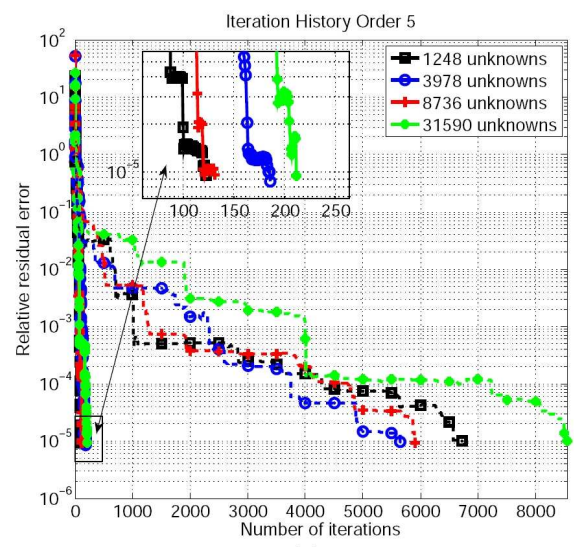

(e)

Fig. 8. Residual history of diagonally-preconditioned EFIE (dashed lines) and HO-CMP (solid lines) for the case of a PEC cube of side $1 \mathrm{~m}$., illuminated by a $30 \mathrm{MHz}$., $\hat{x}$-polarized plane wave traveling in the $\hat{z}$ direction. Four different discretizations are used, ranging from 24 to 918 elements. Results are shown for several orders of the basis functions: (a) order 1; (b) order 2; (c) order 3; (d) order 4; (e) order 5. 


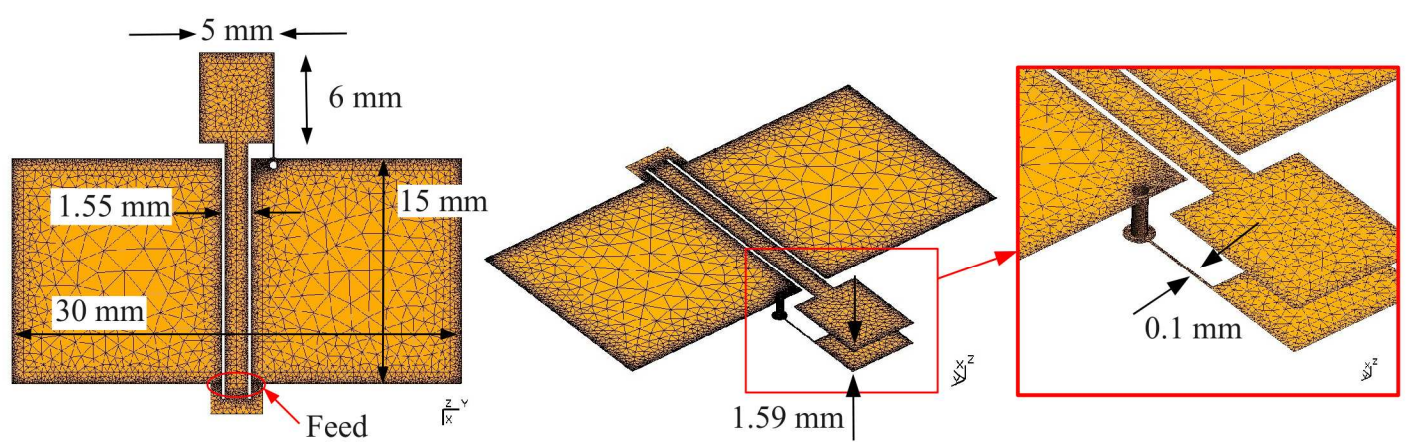

(a)
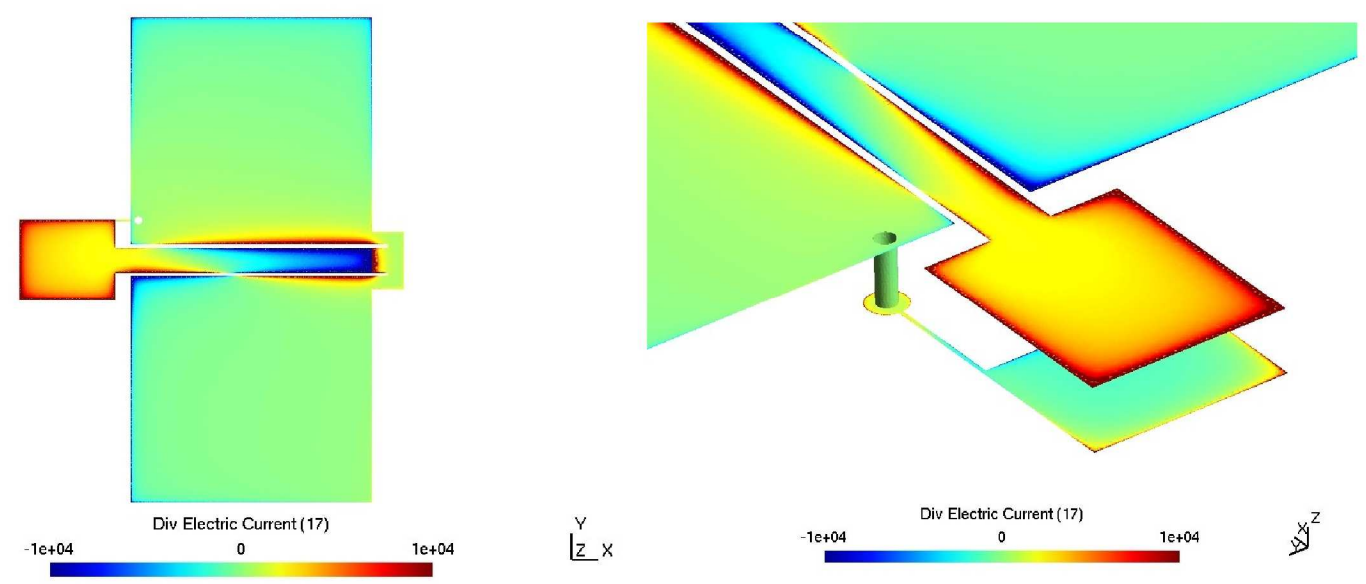

$\underline{Z} \times$

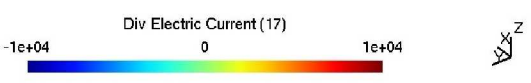

(b)

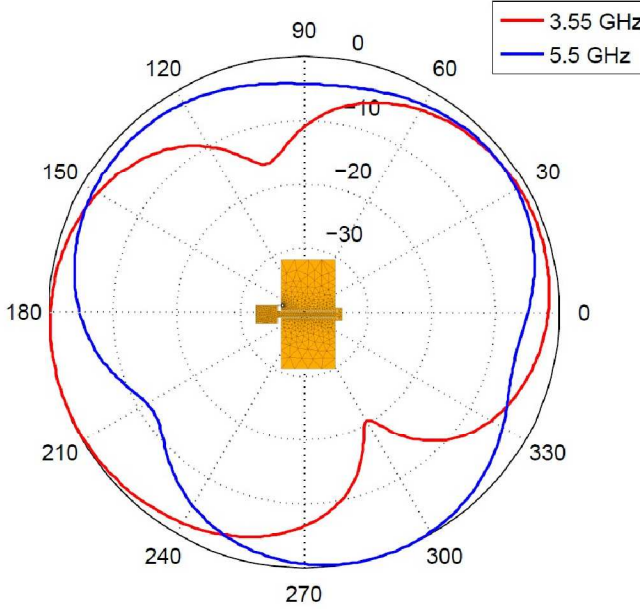

(c)

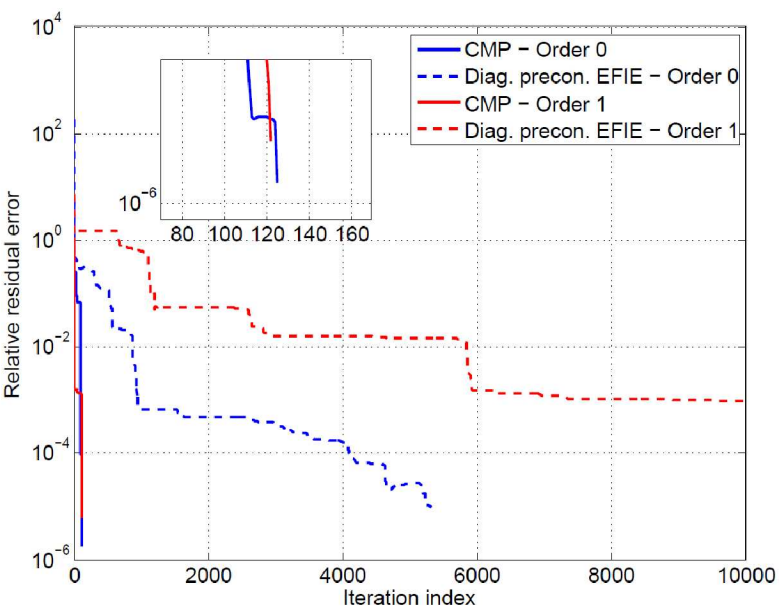

(d)

Fig. 9. Monopole antenna excited with a voltage delta-gap. (a) Mesh and dimensions of the antenna. (b) Divergence of the current density induced on the antenna, for a frequency of $3.55 \mathrm{GHz}$. (c) Radiation pattern in the x-y plane for two different frequencies. (d) Residual history of diagonallypreconditioned EFIE (dashed lines) and HO-CMP (solid lines), for a frequency of $5.5 \mathrm{GHz}$ for orders $p=0,1$. 


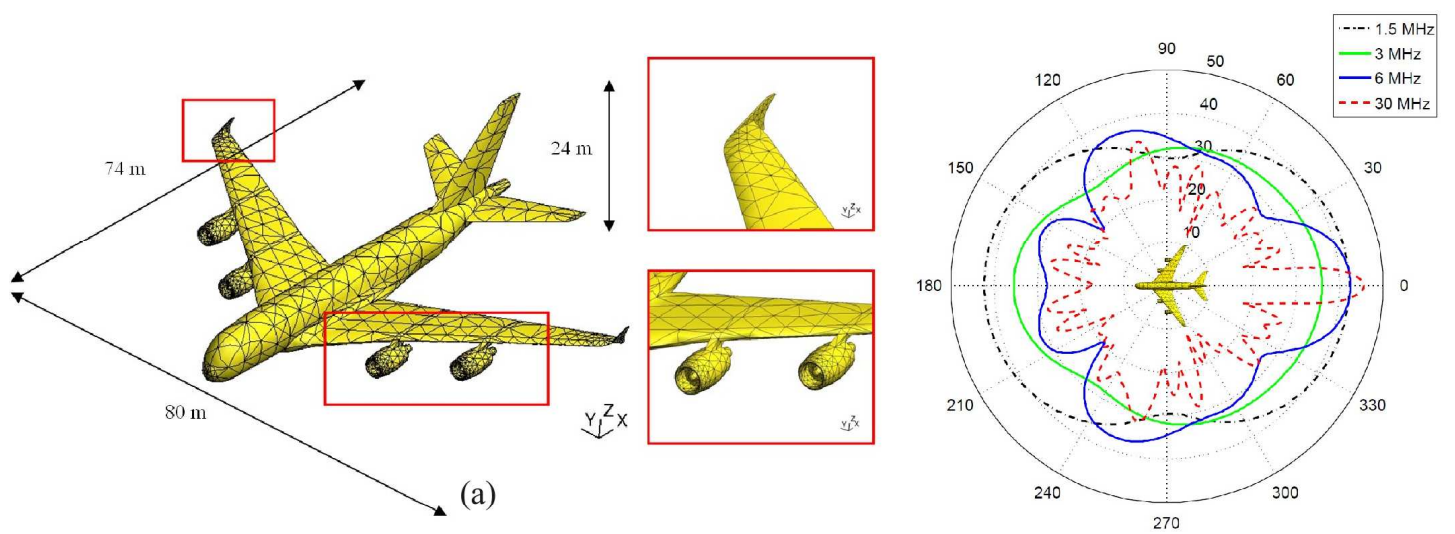

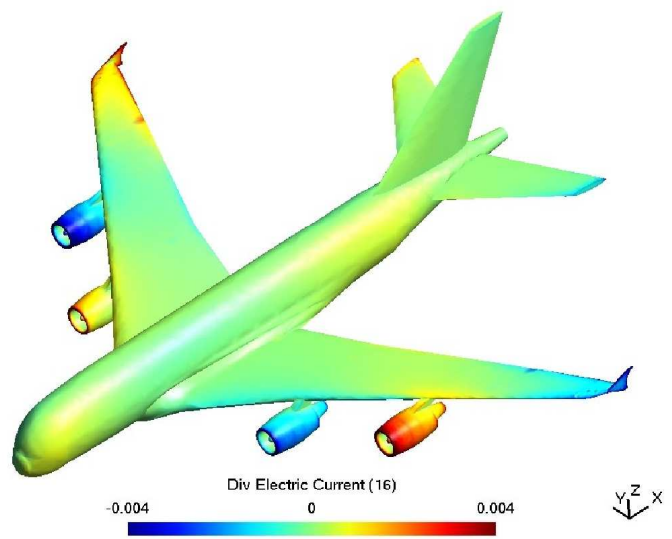

(c)

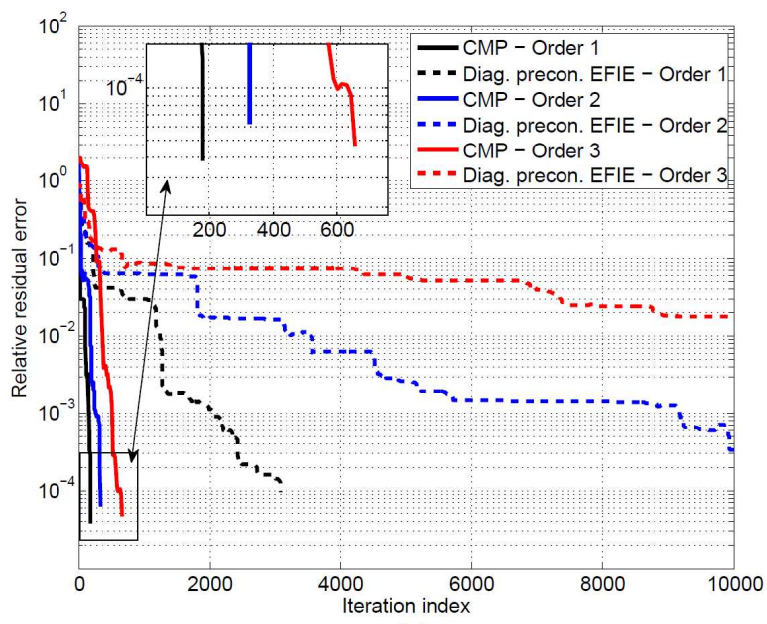

(e)

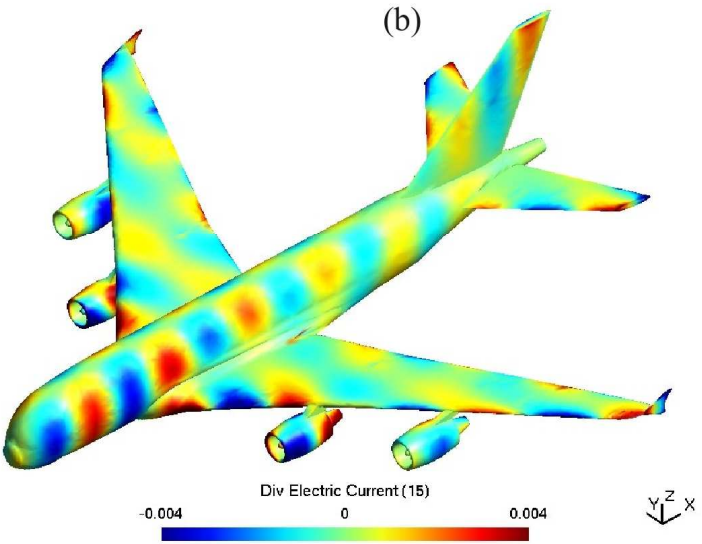

(d)

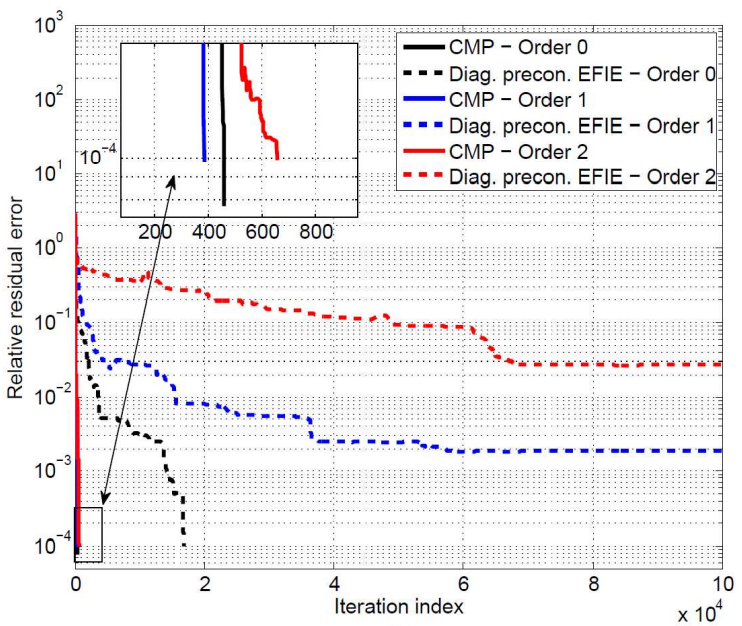

(f)

Fig. 10. Airbus A380 model illuminated by $\hat{\boldsymbol{y}}$-polarized plane wave traveling in the $\hat{\boldsymbol{x}}$ direction. (a) Mesh and dimensions of the aircraft; second order curvilinear patches are used to discretize the surface. (b) Bistatic RCS in the x-y plane for four different frequencies. (c) Divergence of the current density induced on the aircraft, for a frequency of $6 \mathrm{MHz}$. (d) Divergence of the current density induced on the aircraft, for a frequency of $30 \mathrm{MHz}$. (e) Residual history of diagonally-preconditioned EFIE (dashed lines) and HO-CMP (solid lines), for a frequency of 6 MHz for orders $p=1,2,3$. (f) Residual history of diagonally-preconditioned EFIE (dashed lines) and HO-CMP (solid lines), for a frequency of $30 \mathrm{MHz}$ for orders $p=0,1,2$. 